\title{
Modelo de gestión de contratistas para mejorar las condiciones de seguridad y productividad en una empresa del sector industrial
}

Franco Rojas Albán ${ }^{1}$

\begin{abstract}
RESUMEN
La contratación de servicios de terceros contribuye al desarrollo del negocio. En algunas ocasiones, para ganar licitaciones a bajo costo, los contratistas aceptan realizar trabajos en condiciones inseguras; en otras, el cliente presiona para que se complete el servicio antes del plazo establecido, por lo que los contratistas toman "atajos" y evaden controles de seguridad, lo que aumenta el riesgo de lesiones graves, enfermedades crónicas o incluso la muerte. En el Perú, no existe un modelo de gestión de contratistas que mejore las condiciones de seguridad y promueva la productividad de terceros. Por medio de pruebas estadísticas y de correlación de variables, en esta investigación se ha desarrollado e implementado un modelo de gestión que describe los requisitos mínimos que se deben cumplir para evitar incidentes y mejorar la productividad de los contratistas. Este incluye normativas de reconocidas agencias nacionales e internacionales e integra las mejores prácticas de trabajo adquiridas en los más de 30 años de experiencia en el manejo de incidentes de manufactura, construcción, acería y minera.
\end{abstract}

Palabras clave: gestión de contratistas; seguridad en el trabajo de terceros; productividad para contratistas.

\section{INTRODUCCIÓN}

Esta investigación aporta un modelo de gestión diseñado para los proveedores de servicios (contratistas) y organizaciones que contratan servicios de terceros (clientes). Este modelo permite:

- Proteger al personal contratista de lesiones y enfermedades causadas por las actividades del cliente

- Salvaguardar los activos de ambos partes interesadas

- Evitar la pérdida de productividad por lesiones o enfermedades de trabajadores contratistas

- Evitar la inflación de los costos y los plazos de entrega de proyectos o servicios

- Mejorar el bienestar del contratista, imagen social, opinión pública y confianza en el mercado

- Esclarecer la responsabilidad legal ante la investigación de accidentes laborales o inspectorías del Estado

- Evitar el incremento de la siniestralidad y los sobrecostos del seguro

- Mejorar de forma continua el desempeño y promover una cultura de trabajo seguro

La presente investigación es relevante y novedosa para el sector manufactura peruano, ya que no existe un estándar o modelo de gestión de la seguridad y productividad de los contratistas que profundice más allá de lo requerido por la legislación peruana con respecto al trabajo de terceros. El objetivo del presente artículo, así como su hipótesis, es demostrar que la implementación de un modelo de gestión de contratistas (MGC) mejora las condiciones de seguridad y la productividad de terceros en una empresa del sector industrial.

Esta investigación se llevó a cabo en una empresa metalmecánica del sector manufactura, la cual transforma el acero en consumibles para la industria minera. Dicha empresa cuenta con

1 Ingeniero industrial por la Universidad Nacional de San Agustín de Arequipa. Actualmente, es ingeniero de seguridad de la empresa Yura S.A. (Arequipa, Perú). ORCID: https://orcid.org/0000-0001-8628-4793

E-mail: frojas87@gmail.com 
tres plantas industriales distribuidas en el Perú. El nombre de la empresa y de los productos finales se mantendrán en reserva por motivos de seguridad y confidencialidad; para fines de esta investigación, se hará referencia a la empresa analizada con el nombre de "RODAS S.A."

Los resultados de esta investigación pueden extenderse a otras organizaciones del sector manufactura, dada la gran similitud en la forma de gestionar las actividades de la industria en el periodo de estudio. En los siguientes párrafos se muestra esa similitud en cuanto a la contratación de servicios terceros y los accidentes laborales del sector.

En 2018, la Asociación de Empresas de Tercerización y Trabajo Temporal del Perú (AETT Perú) y el Corporativo Overall indicaron que 8 de cada 10 empresas en el Perú optaban por la tercerización de servicios (Diario Gestión, 2018), entre los que se encuentran: reclutamiento, alimentación, payroll, transporte, vigilancia, limpieza, gasfitería, albañilería, evacuación de residuos, mantenimiento, construcción, etc. "Tres sectores encabezan la tercerización: Minería y Petróleo con el $50 \%$, Servicio Retail y Consumo con el $30 \%$ y Otros (Agricultura, Construcción, Manufactura) con el 20\%" (Diario Gestión, 2015, párr. 1).

En su "Anuario estadístico sectorial 2016", el Ministerio del Trabajo y Promoción del Empleo (MTPE) informó que durante todo el año se registraron 20 876 accidentes laborales, $17084(81.8 \%)$ de los cuales ocurrieron en Lima y Callao, lo que la convierte en la región con más casos (MTPE, 2017a). Sin embargo, la cantidad de notificaciones de accidentes laborales reportados al MTPE es una cifra subestimada, puesto que muchas empresas formales, pero especialmente las informales, no notifican este tipo de ocurrencias debido al miedo de afrontar sanciones graves impuestas por la SUNAFIL por el incumplimiento de las condiciones de seguridad (Ministerio de Energía y Minas [MINEM], 2020). La Tabla 1 resume dicha estadística para el propósito de este estudio.

\section{Seguridad y salud en el trabajo (SST)}

La seguridad y salud en el trabajo es un aspecto fundamental del trabajo decente. El trabajo decente es un trabajo seguro. Todos los trabajadores deben estar seguros en sus lugares de trabajo, deben tener la tranquilidad de saber que no están expuestos a riesgos ni peligros. Las condiciones físicas y las exigencias mentales del trabajo y del entorno laboral en general tienen un fuerte impacto en el bienestar y las condiciones de vida de los trabajadores. Los accidentes y enfermedades profesionales tienen un importante costo humano, social y económico, por lo que debemos esforzarnos para prevenirlos; para ello, se debe garantizar que todos los lugares de trabajo sean seguros (International Labour Organization [ILO], 2020).

"La salud laboral se construye en un medio ambiente de trabajo adecuado, con condiciones de trabajo justas, donde los trabajadores puedan desarrollar una actividad con dignidad y donde sea posible su participación para la mejora de las condiciones de salud y seguridad". (Instituto Sindical de Trabajo, Ambiente y Salud [ISTAS], s.f., párr. 1)

Según la OIT y la OMS, la salud ocupacional es

la promoción y mantenimiento del mayor grado de bienestar físico, mental y social de

Tabla 1. Ranking comparativo de accidentes de trabajo y sus tipos.

\begin{tabular}{|c|c|c|c|}
\hline Accidentes por actividad económica (Top 3) & $\%$ & Consecuencias del accidente & $\%$ \\
\hline Industrias manufactureras & $25 \%$ & Accidentes leves & $53 \%$ \\
\hline Actividades inmobiliarias, empresariales y de alquiler & $19 \%$ & Accidentes incapacitantes & $46 \%$ \\
\hline Construcción & $11 \%$ & Accidentes mortales & $1 \%$ \\
\hline $\begin{array}{l}\text { Accidentes por ocupación } \\
\text { (Top 3) }\end{array}$ & $\%$ & $\begin{array}{c}\text { Enfermedades ocupacionales por actividad } \\
\text { económica (Top 3) }\end{array}$ & $\%$ \\
\hline No especificado* & $32 \%$ & Explotación de minas y canteras & $59 \%$ \\
\hline Operario & $27 \%$ & Industrias manufactureras & $13 \%$ \\
\hline Otras & $18 \%$ & Servicios sociales y de salud & $9 \%$ \\
\hline
\end{tabular}

*Incluye a trabajadores u operarios tercerizados.

Fuente: Anuario MTPE (2017a). 
los trabajadores en todas las ocupaciones mediante la prevención de las desviaciones de la salud, control de riesgos y la adaptación del trabajo a la gente, y la gente a sus puestos de trabajo. (Organización Panamericana de la Salud [OPS], 2020, párr. 1)

La salud ocupacional no se limita a resguardar el bienestar físico del trabajador, sino que también se ocupa del aspecto psicológico. Para los empleadores, la salud ocupacional supone un apoyo al perfeccionamiento del trabajador y al mantenimiento de su capacidad de trabajo (OPS, 2020).

\section{Indicadores de SST y productividad}

1. Índice de frecuencia de incidentes con lesión (IFIC). Según la OIT, el también llamado índice de accidentabilidad se obtiene de la división entre el número de incidentes laborales con lesión ocurridos durante el periodo de estudio y las horas hombre del grupo de referencia. La relación se multiplica por un número de ajuste, el cual, para su mejor interpretación, se ajusta según la magnitud de las horas trabajadas, las cuales pueden ser 100, 1000, 100000 , 1000 000, etc. (ILO, 2020).

$I F=\frac{\text { Incidentes con lesión del periodo estudiado }}{H H} \times 10^{5}$

2. Índice de frecuencia de incidentes sin lesión (IFIS). Este índice se obtiene para validar los efectos presentados en el desarrollo del MGC.

$I F=\frac{\text { Incidentes sin lesión del periodo estudiado }}{H H} \times 10^{5}$

Es importante indicar que las horas hombre $(\mathrm{HH})$ se obtienen del producto entre el número de trabajadores del grupo de referencia y el número de horas trabajadas por los mismos (ILO, 2020). En este caso, el grupo de referencia está conformado por los trabajadores contratistas.

$H H=$ Cantidad de trabajadores contratistas $\times$ Total de horas trabajadas

3. Disponibilidad de tiempo para el servicio (DTS). De manera semejante a los indicadores de bienes y productos, el tiempo disponible para brindar un servicio tiene la siguiente fórmula:

$$
\text { DTS }=\frac{\text { Tiempo ef ectivo }}{\text { Tiempo disponible }} \times 100
$$

Donde el tiempo disponible es el tiempo asignado por la organización que contrata el servicio (cliente) y el tiempo efectivo es el tiempo disponible menos el tiempo de paradas no programadas que hayan sucedido por interrupciones del servicio como incidentes, demoras en entrega de material, equipos, herramientas defectuosas, otras actividades o prioridades, etc.

Tiempo efectivo $=T$. disponible $-T$. paradas no programadas

Tiempo disponible $=$ Tiempo asignado para el servicio

\section{Modelo de gestión de contratistas (MGC)}

Este modelo está elaborado para ser utilizado por las diferentes compañías del sector industrial, ya sea manufactura, acería, construcción o minería, independientemente de su tamaño.

El proceso inicia cuando el plan de trabajo aprobado de un proyecto o servicio incluye contratistas de forma parcial o total. En la Figura 1, se muestran los 4 principales subprocesos del MGC:

En la Figura 2, se muestran las etapas del proceso para definir las condiciones de seguridad y salud en el trabajo (SST) que se deben cumplir para realizar las actividades del plan aprobado; acto seguido, se identifica el nivel de riesgo asociado a cada actividad para así obtener como resultado los requisitos básicos y específicos, según su complejidad, para que la organización pueda licitar el servicio o proyecto con contratistas en igualdad de condiciones.

Una vez recibidas las propuestas, se procede a realizar su evaluación (Figura 3). Si el contratista trabajó antes para la organización, se revisan los registros de la evaluación integral, se elabora una tabla comparativa y se selecciona al contratista con mayor puntaje para la realización del proyecto o servicio:

Antes de iniciar el trabajo, el contratista seleccionado debe pasar por un proceso de inducción a la cultura SST de la organización, lo que incluye las capacitaciones en trabajos de alto riesgo requeridos en el plan. Tras aprobar el examen de conocimientos de la inducción, se les genera una validación de ingreso personal (ver Figura 4).

Con la autorización de ingreso vigente, se revisan y validan el resto de requisitos de SST (MTPE, 2017b), como el seguro complementario de trabajo de riesgo (SCTR), el apto médico, la inspección de herramientas de trabajo y demás documentos de seguridad requeridos por el cliente. El proceso de 


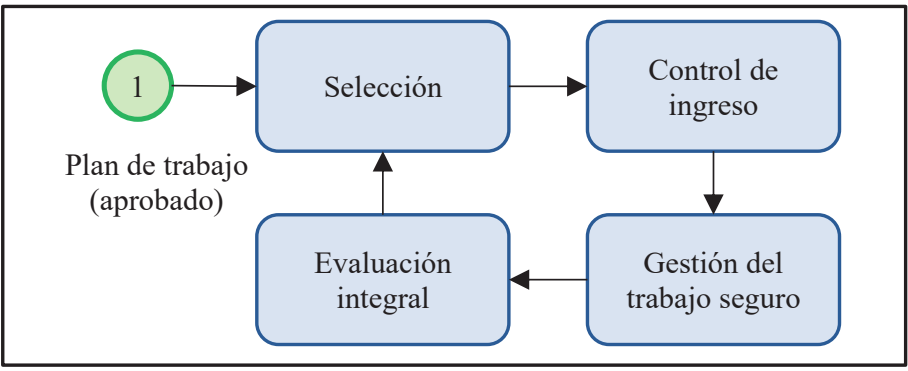

Figura 1. Diagrama general del sistema de gestión de contratistas. Fuente: Elaboración propia.

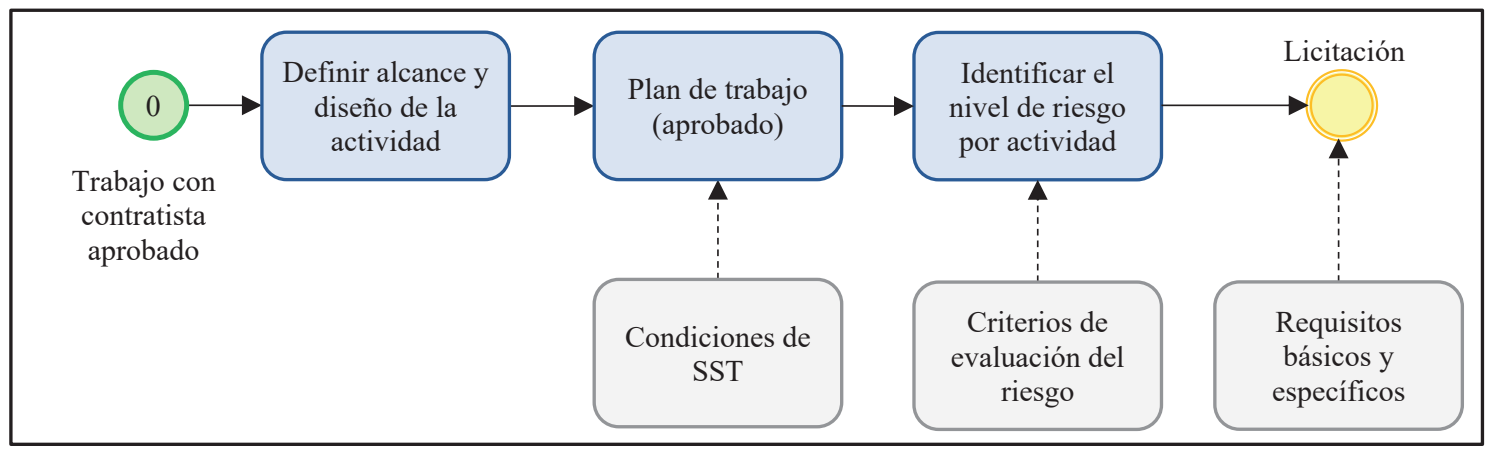

Figura 2. Plan de trabajo para contratistas. Fuente: Elaboración propia.

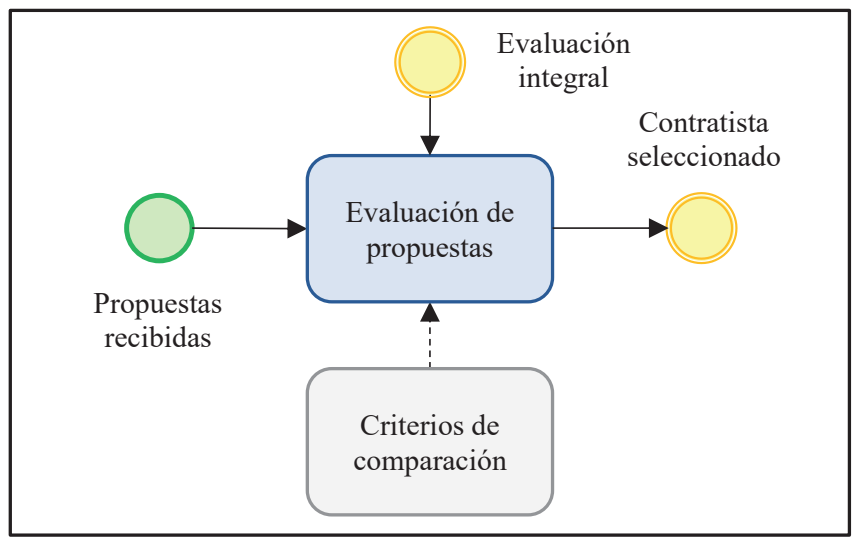

Figura 3. Evaluación y selección de contratistas.

Fuente: Elaboración propia.

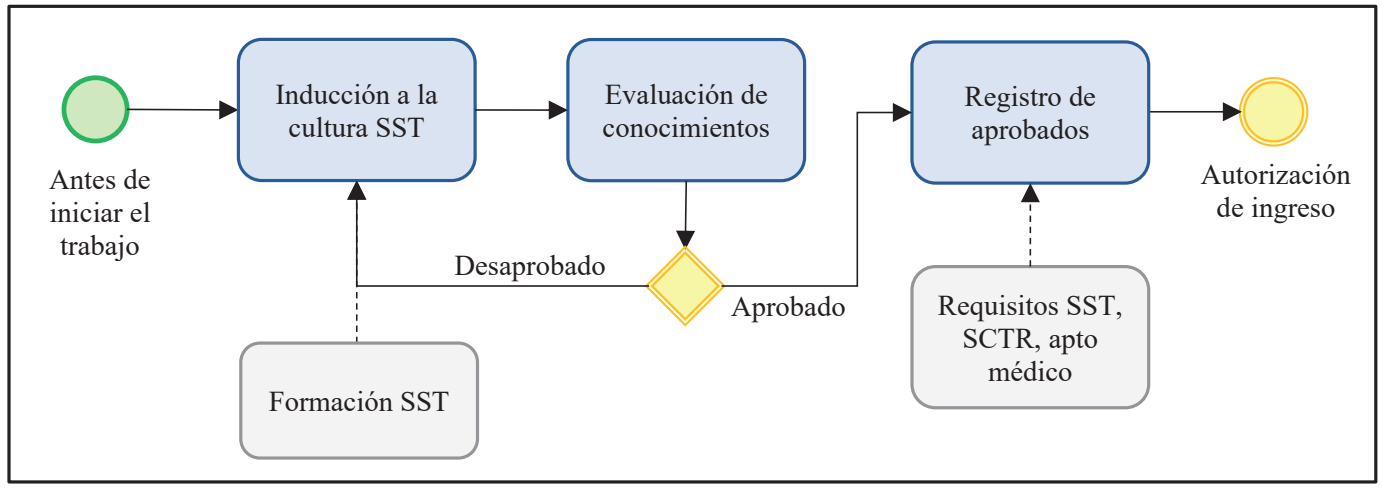

Figura 4. Autorización de ingreso al contratista al centro de trabajo.

Fuente: Elaboración propia. 
autorización de trabajo está a cargo del supervisor de tareas asignado por el cliente. Toda inspección, observación, accidente laboral o evento de SST es registrado en el MGC (ver Figura 5).

Una vez finalizado el trabajo, el contratista es evaluado de forma integral tomando como base los criterios e indicadores preestablecidos en el MGC (ver Figura 6).

\section{METODOLOGÍA}

Con base en el libro "Metodología de la Investigación” de Hernández, Collado, y Baptista (2010), se determinó que el alcance de la presente investigación es de tipo correlacional, ya que tiene como finalidad conocer la relación o grado de asociación que existe entre la variable independiente X, "Implementación del modelo gestión de contratistas", y dos variables dependientes: Y, "Condiciones de seguridad", y Z, "Productividad", en el contexto de una empresa del sector industrial.

Mediante auditorías internas, se midió trimestralmente el porcentaje de implementación del MGC (\% Imp. MGC). Este porcentaje refleja el porcentaje de cumplimiento del momento, por lo que puede variar en el tiempo, aunque conservando una mar-

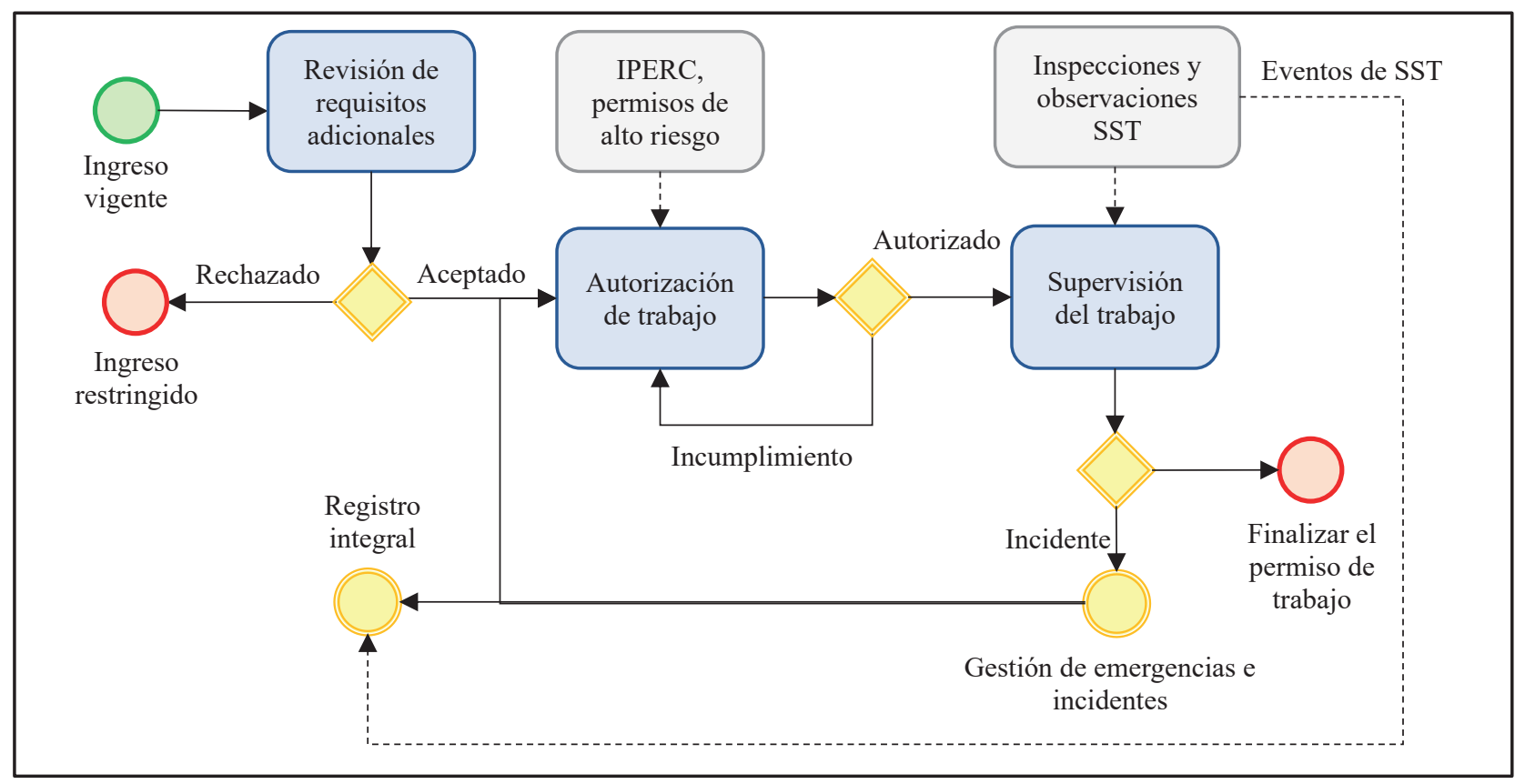

Figura 5. Gestión del trabajo seguro.

Fuente: Elaboración propia.

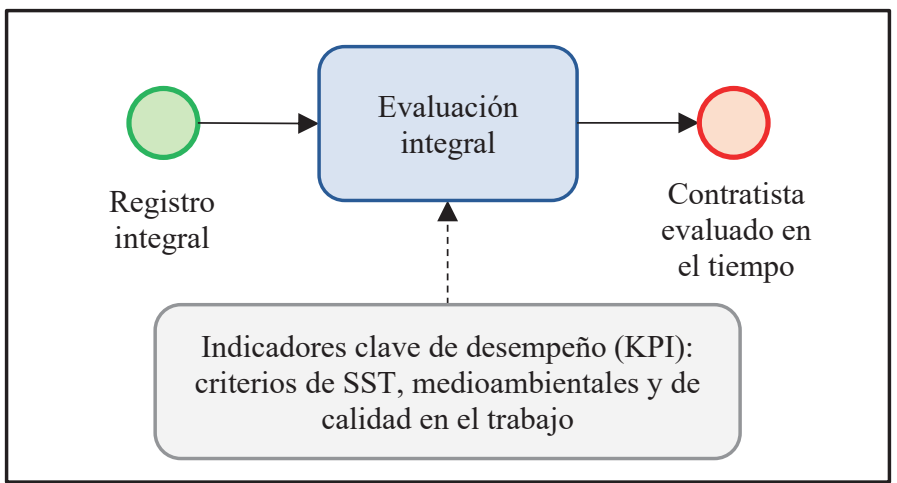

Figura 6. Evaluación integral del contratista considerando criterios de SST, calidad y medio ambiente.

Fuente: Elaboración propia. 
cada tendencia. Se midieron los cambios observados en las condiciones de seguridad (frecuencia de incidentes) y productividad (disponibilidad de tiempo del servicio) utilizando las unidades de análisis recolectadas durante el periodo 2012 - 2016.

Se demostró que la correlación entre variables es causal, es decir, que la mejora observada en las variables dependientes $Y$ y $Z$ resultó de la mejora en la variable independiente $X$. En la Tabla 2 se muestra dicha relación.

"Una población es el conjunto de todos los casos que concuerdan con una serie de especificaciones" (Selltiz, 1980, citado por Hernández, 2010, p. 65). En este estudio, se tomó el $100 \%$ de los datos generados en el periodo 2012 - 2016 de la empresa Rodas S.A. en relación al porcentaje de implementación del modelo de gestión de contratistas, la cantidad de horas hombres trabajadas, los incidentes de sus contratistas y el tiempo de paradas desde el incidente hasta que el cliente determinara que era seguro continuar.

La recolección de datos se realizó con base en el estándar y criterios de la OIT establecidos en la publicación "Statistics of Occupational Injuries, Sixteenth International Conference of Labour Statisticians" (ILO, 1998), ahora actualizados en la "Quick guide on sources and uses of statistics on occupational safety and health" (ILO, 2020). El método usado se describe en la Tabla 3.

Siguiendo las recomendaciones de la Organización Internacional del Trabajo, se separaron estadísticamente los incidentes fatales de los no fatales, con el fin de evitar datos sesgados y/o valores que se alejan de forma excesiva de la tendencia y distribución de los valores (ILO, 2020).

En la Tabla 4 se muestra el resumen de horas hombre e incidentes con lesión y sin lesión ocurridos por año.

En la Tabla 5 se resume el tiempo de parada por incidentes por año:

La Tabla 6, resume el porcentaje de implementación del MGC por año, hallado cada tres meses utilizando el formato de auditoría en el Anexo 01:

\section{RESULTADOS}

Para interpretar y evaluar los resultados es importante entender las propiedades estadísticas descriptivas o inferenciales de los datos resultantes. A continuación, se muestra el resumen de la estadística descriptiva de los indicadores de las variables en la Tabla 7.

Tabla 2. Unidad de análisis y medición.

\begin{tabular}{|l|l|l|}
\hline \multicolumn{1}{|c|}{ Variable } & \multicolumn{1}{|c|}{ Unidad de análisis } & \multicolumn{1}{c|}{ Medición } \\
\hline $\begin{array}{l}\text { Modelo de gestión de } \\
\text { contratistas }(\mathrm{X})\end{array}$ & 1. Porcentaje de implementación & $\begin{array}{l}\text { - Trimestral, según resultado de auditorías - } \\
\text { Anexo } 01\end{array}$ \\
\hline Condiciones de seguridad $(\mathrm{Y})$ & $\begin{array}{l}\text { Indicadores de SST: } \\
\text { 2. Índice de frecuencia de incidentes sin lesión } \\
\text { IFIS } \\
\text { 3. Índice de frecuencia de incidentes con lesión } \\
\text { IFIC }\end{array}$ & $\begin{array}{l}\text { - Incidentes con y sin lesión } \\
\text { - Cantidad de horas hombre de trabajadores } \\
\text { contratistas }\end{array}$ \\
\hline Productividad $(\mathrm{Z})$ & 4. Disponibilidad de tiempo para el servicio & $\begin{array}{l}\text { - Tiempo de parada del servicio de contratistas } \\
\text { por incidentes }\end{array}$ \\
\hline
\end{tabular}

Fuente: Elaboración propia.

Tabla 3. Método para la recolección de datos.

\begin{tabular}{|c|c|c|c|}
\hline Var. & Medición & \multicolumn{1}{c|}{ Método para la recolección } \\
\hline $\mathrm{X}$ & $\begin{array}{c}\text { Trimestral, según resultado } \\
\text { de auditorías }\end{array}$ & $\begin{array}{l}\text { Para llevar a cabo la auditoría al MGC, es necesario mostrar documentación, realizar observacio- } \\
\text { nes de campo, entrevistas y encuestas que puedan ser constatadas. El modelo de esta auditoría } \\
\text { está en el Anexo 01. }\end{array}$ \\
\hline Y & $\begin{array}{c}\text { Cantidad de horas hombre } \\
\text { de trabajadores contratistas } \\
\text { e incidentes con y sin lesión }\end{array}$ & $\begin{array}{l}\text { Se requiere la medición, el análisis y la evaluación del 100\% de los datos recolectados durante el } \\
\text { periodo 2012 - 2016 en la empresa Rodas S. A. Dado que es un análisis de variables puramente } \\
\text { cuantitativo, no es necesario realizar entrevistas ni encuestas. }\end{array}$ \\
\hline Z & $\begin{array}{c}\text { Tiempo de parada del } \\
\text { servicio de contratistas por } \\
\text { incidentes }\end{array}$ & $\begin{array}{l}\text { Se requiere la medición, el análisis y la evaluación del 100\% de los datos recolectados durante el } \\
\text { periodo 2012 - 2016 en la empresa Rodas S. A. }\end{array}$ \\
\hline
\end{tabular}

Fuente: Elaboración propia. 
Tabla 4. Cantidad de incidentes de trabajadores contratistas por año.

\begin{tabular}{|c|c|c|c|c|}
\hline Año & $\begin{array}{c}\text { Trabajadores contratistas } \\
\text { (máx.) }\end{array}$ & $\begin{array}{c}\text { Horas hombre } \\
\text { (máx.) }\end{array}$ & Incidentes sin lesión & Incidentes con lesión \\
\hline 2012 & 273 & 78656 & 2 & 23 \\
\hline 2013 & 325 & 86625 & 5 & 19 \\
\hline 2014 & 362 & 101424 & 16 & 9 \\
\hline 2015 & 427 & 119236 & 25 & 5 \\
\hline 2016 & 553 & 146739 & 17 & 60 \\
\hline \multicolumn{2}{|r|}{ Total de incidentes } \\
\hline
\end{tabular}

Fuente: Elaboración propia con base en la información proporcionada por la empresa

Tabla 5. Resumen de paradas del servicio por año.

\begin{tabular}{|c|c|}
\hline Año & Parada del servicio (hrs.) \\
\hline 2012 & 170.38 \\
\hline 2013 & 165.73 \\
\hline 2014 & 66.93 \\
\hline 2015 & 25.08 \\
\hline 2016 & 33.41 \\
\hline Total general & $\mathbf{4 6 1 . 5 3}$ \\
\hline
\end{tabular}

Fuente: Elaboración propia con base en la información proporcionada por la empresa

Tabla 6. Resumen del porcentaje de implementación del MGC.

\begin{tabular}{|c|c|}
\hline Año & Implementación del MGC (\%) \\
\hline $\mathbf{2 0 1 2}$ & $7 \%$ \\
\hline $\mathbf{2 0 1 3}$ & $15 \%$ \\
\hline $\mathbf{2 0 1 4}$ & $87 \%$ \\
\hline $\mathbf{2 0 1 5}$ & $99 \%$ \\
\hline $\mathbf{2 0 1 6}$ & $95 \%$ \\
\hline
\end{tabular}

Fuente: Elaboración propia.

Tabla 7. Estadística descriptiva de los indicadores de las variables.

\begin{tabular}{|l|c|c|c|}
\hline & IFIS & IFIC & DTS \\
\hline Media & 1.32 & 1.67 & 7.69 \\
\hline Mediana & 1.51 & 1.19 & 4.37 \\
\hline Moda & 0.00 & 0.00 & 0.00 \\
\hline Desviación estándar & 1.15 & 1.83 & 9.22 \\
\hline Varianza de la muestra & 1.32 & 3.35 & 85.07 \\
\hline Curtosis & -1.05 & 0.62 & 4.94 \\
\hline Coeficiente de asimetría & 0.29 & 1.10 & 1.84 \\
\hline
\end{tabular}

Fuente: Elaboración propia.

Los valores de la media y la mediana son cercanos en el caso de IFIS e IFIC; sin embargo, los valores de la moda, en el caso de todos los indicadores, indica que probablemente la distribución no sea normal. Para corroborar que los datos están distribuidos de forma no uniformes, se aplicó la prueba Shapiro Wilk que es utilizada para contrastar la normalidad de un conjunto de datos (Shapiro y Wilk, 1965). Se plantea como hipótesis nula $\left(\mathrm{H}_{0}\right)$ que una muestra $\mathrm{x}_{1}, \ldots, \mathrm{x}_{\mathrm{n}}$ proviene de una población con distribución normal (ver Tabla 8).

La desviación estándar y la varianza de los valores de IFIS muestran que la dispersión es cercana; por 
el contrario, el resto de variables indican alta variabilidad para los promedios con respecto a la tendencia de la muestra. La curtosis y el coeficiente de asimetría dan una idea de cómo está formada la curva de distribución, la prominencia y simetría de sus colas (Minitab, 2019).

Con base en los resultados de las pruebas de normalidad, se utilizó la prueba de correlación de Spearman, la cual se aplica para evaluar relaciones en las que intervienen variables ordinales con distribuciones no lineales (Zar, 1984).

En la Figura 7 se muestra un resumen de los incidentes ocurridos por año para comparar la evolución entre los incidentes con lesión y sin lesión, y su interacción con la implementación del MGC. Se aprecia que, en los dos primeros años del estudio, la cantidad de incidentes con lesión es significativamente alta con respecto al porcentaje de implementación del MGC; por el contrario, sucede lo opuesto en los dos últimos años del estudio. Se puede inferir entonces que hay una relación proporcional inversa o negativa entre las variables.
En la Figura 8 se agrupa el promedio anual del IFIS para comparar su evolución en el tiempo con respecto al porcentaje de implementación del MGC. Se aprecia que, durante los dos primeros años del estudio, tanto el IFIS como el porcentaje de implementación del son significativamente bajos; por el contrario, son significativamente altos en los dos años siguientes. Se puede inferir entonces que hay una relación proporcional directa o positiva entre las variables.

En la Figura 9 se agrupa el promedio anual del IFIC para comparar su evolución en el tiempo con respecto al porcentaje de implementación del MGC. Se aprecia que, durante los dos primeros años del estudio, el IFIC es significativamente alto y el porcentaje de implementación del MGC es bajo; por el contrario, sucede lo opuesto en los dos últimos años del estudio. Se puede inferir entonces que hay una relación proporcional inversa entre las variables.

En la Figura 10 se agrupa y calcula el promedio anual de la DTS para comparar su evolución en el tiempo con respecto al porcentaje de implementación del

Tabla 8. Pruebas de normalidad.

\begin{tabular}{|c|c|c|c|c|}
\hline Indicador & Shapiro-Wilk (W) & $\begin{array}{c}\boldsymbol{p} \text {-valor } \\
\mathbf{( 0 . 0 5 )}\end{array}$ & Evaluación $\left.\mathbf{( H}_{\mathbf{0}}\right)$ & Resultado \\
\hline \% Imp. MGC & 0.7324 & $3.94 \mathrm{e}-09$ & Rechazada & Distribución no normal \\
\hline IFIS & 0.8901 & $5.82 \mathrm{e}-05$ & Rechazada & Distribución no normal \\
\hline IFIC & 0.8473 & $2.54 \mathrm{e}-06$ & Rechazada & Distribución no normal \\
\hline DTS & 0.7852 & $5.88 \mathrm{e}-08$ & Rechazada & Distribución no normal \\
\hline
\end{tabular}

Fuente: Elaboración propia.

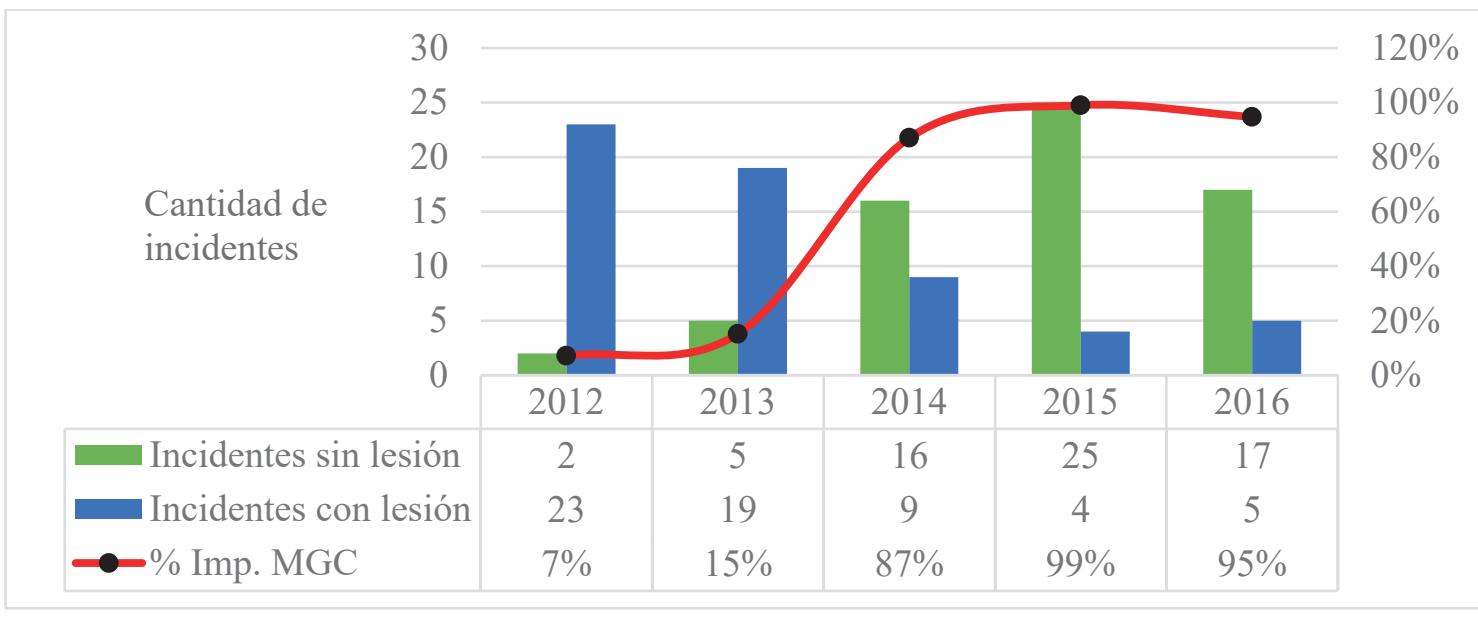

Figura 7. Evolución de incidentes e implementación del MGC.

Fuente: Elaboración propia. 


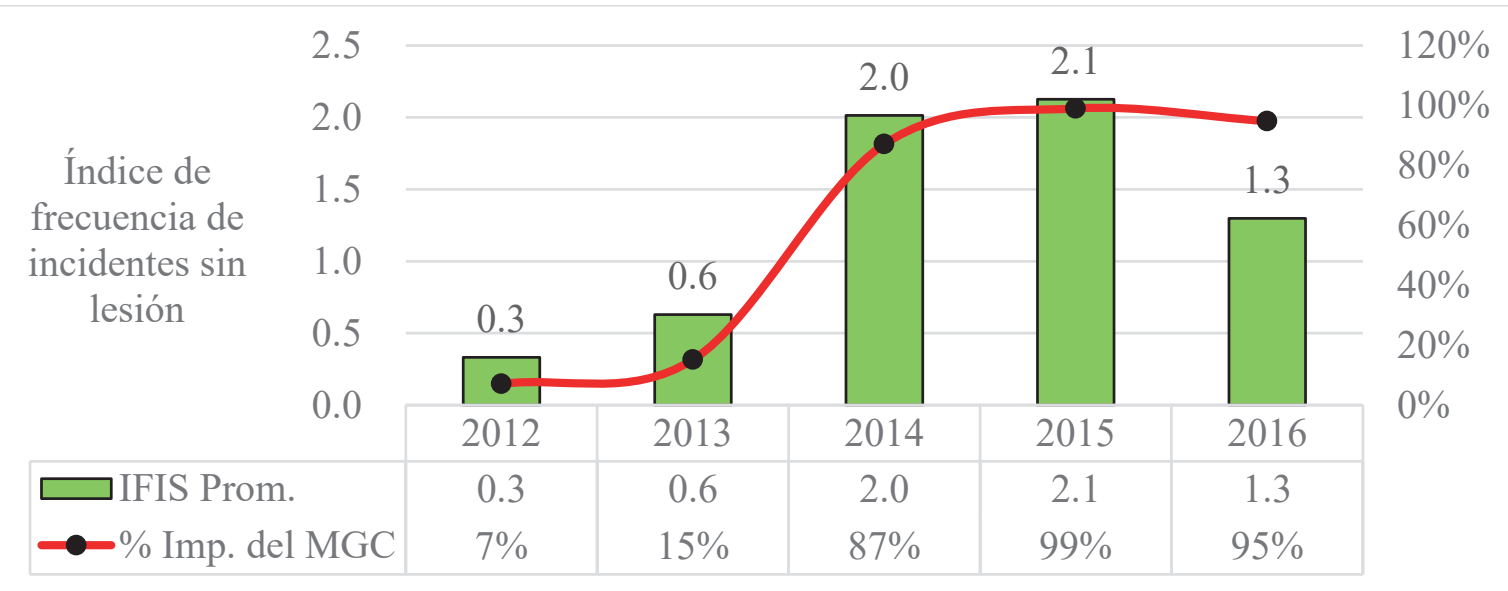

Figura 8. Evolución del IFIS y el porcentaje de implementación del MGC.

Fuente: Elaboración propia.

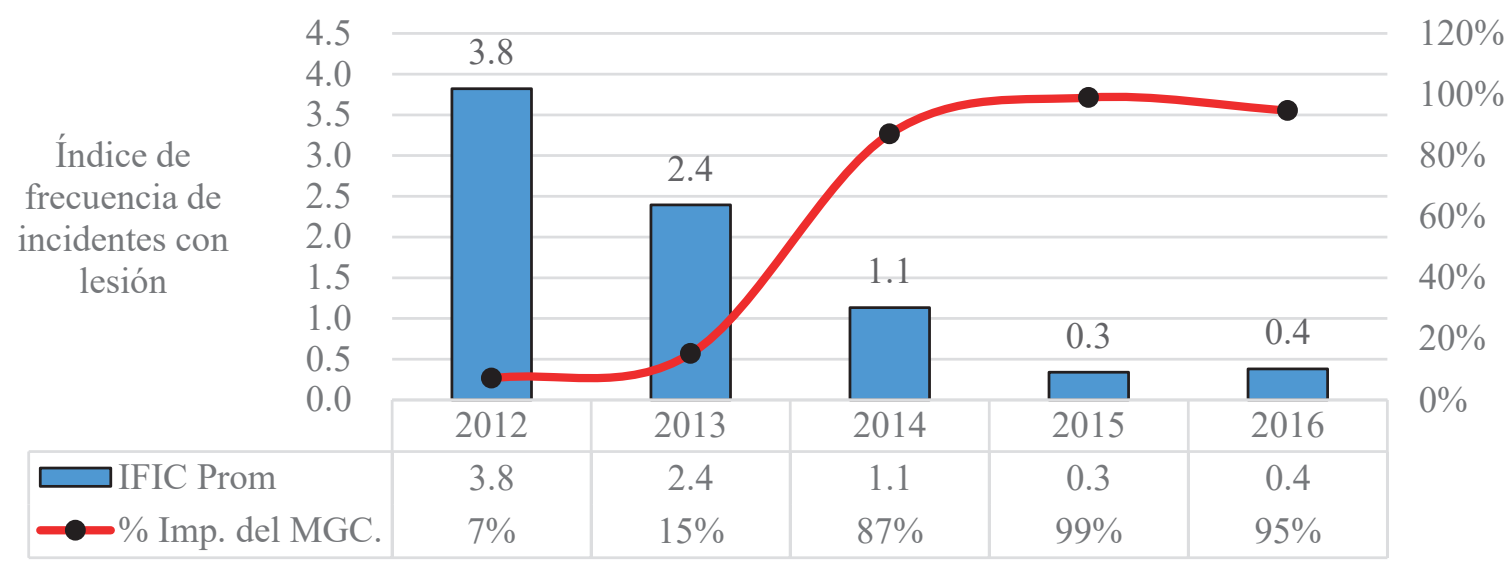

Figura 9. Evolución del IFIC y \% de Implementación del MGC.

Fuente: Elaboración propia.

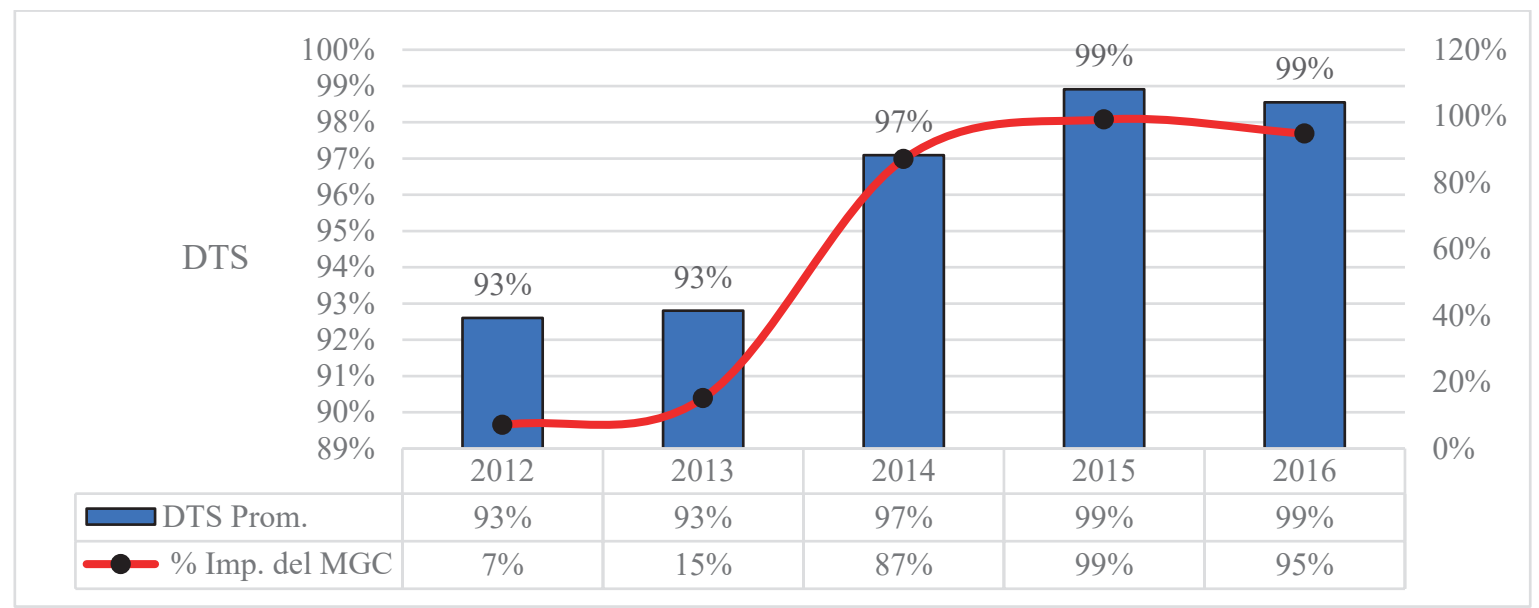

Figura 10. Disponibilidad de tiempo para el servicio y porcentaje implementación del MGC.

Fuente: Elaboración propia. 
MGC. Se aprecia que, durante todos los años del estudio, tanto el DTS como el porcentaje de implementación del MGC varían proporcionalmente en el tiempo. Se puede inferir entonces que hay una relación directa o positiva entre las variables.

\section{DISCUSIÓN}

Para la interpretación de los resultados y contraste con su respectiva hipótesis (evaluación), se utilizó la escala de valoración de Spearman que se presenta en la Tabla 9; mientras que el resultado de esta correlación se observa en la Tabla 10.

La evaluación de la hipótesis general planteada para la presente investigación encontró que la implementación de un modelo de gestión de contratistas influye de forma moderada y fuerte en las condiciones de seguridad y la productividad de terceros en una empresa del sector industrial.

Esta afirmación se sustenta en los resultados de la correlación de Spearman obtenidos para los indicadores de la variable Condiciones de seguridad que señalan que $63 \%$ del IFIS es directamente explicado por el porcentaje de implementación del MGC; mientras que el $71 \%$ del IFIC es inversamente explicado por el porcentaje de implementación del MGC. Por otro lado, para el indicador de la variable Productividad, el $58 \%$ del DTS es directamente explicado por el porcentaje de implementación del MGC. De este modo se observa una correlación moderadamente fuerte entre variables de la hipótesis general.

\section{CONCLUSIONES}

Se cumplió el objetivo de la investigación. Mediante el uso de pruebas de correlación de variables, se ha logrado demostrar que la implementación de un modelo de gestión de contratistas en una empresa del sector industrial ha tenido y tiene un impacto positivo en la mejora de las condiciones de seguridad laboral para sus contratistas.

Tal como se observa en la Figura 7, mediante el análisis de datos y la aplicación de pruebas de correlación entre variables, se ha demostrado que el MGC implementado al $60 \%$ tiene un impacto significativo en la frecuencia de las lesiones de contratistas.

Se demuestra que la implementación del MGC mejora la productividad de las empresas contratistas en función de la disponibilidad del tiempo para realizar el servicio al evitar paradas del servicio por causa de la ocurrencia de incidentes con lesión.

El riesgo de una actividad con personal contratista influye de forma inversamente proporcional al nivel de implementación del modelo de gestión de contratistas, es decir, que el riesgo será alto si la gestión de contratistas es deficiente.

Existe una correlación positiva entre el modelo de gestión de contratistas y la frecuencia de incidentes sin lesión, debido a que el modelo presentado promueve y motiva a los mismos trabajadores, contratistas o no, a reportar todos los incidentes antes de tener contacto con la energía peligrosa que pueda causarles lesiones con daño permanente o mortal.

Tabla 9. Escala Rho (Spearman).

\begin{tabular}{|c|c|}
\hline Rango & Relación \\
\hline $0.00-0.25$ & Escasa o nula \\
\hline $0.26-0.50$ & Débil \\
\hline $0.51-0.75$ & Entre moderada y fuerte \\
\hline $0.76-1.00$ & Entre fuerte y perfecta \\
\hline
\end{tabular}

Fuente: Rangos de Spearman (Martínez, Tuya, Martínez, Pérez y Cánovas, 2009).

Tabla 10. Correlación entre las variables y la implementación del MGC.

\begin{tabular}{|l|c|c|c|c|}
\hline \multicolumn{1}{|c|}{ Variable } & Indicador & Relación & Rho & Evaluación \\
\hline \multirow{2}{*}{ Condiciones de seguridad } & IFIS & Directa & 0.63 & Moderada y fuerte \\
\cline { 2 - 5 } & IFIC & Inversa & 0.71 & Moderada y fuerte \\
\hline Productividad & DTS & Directa & 0.58 & Moderada y fuerte \\
\hline
\end{tabular}

Fuente: Elaboración propia. 
Finalmente, se puede concluir que la implementación de un modelo de gestión de contratistas mejora las condiciones de seguridad y productividad en una empresa del sector industrial.

\section{RECOMENDACIONES}

Para mejorar la implementación del MGC es importante que la alta dirección y las gerencias entiendan, estén capacitados y motivados en implementar el modelo de gestión al 100\%, dado que, sin la asignación de personal y recursos, se pierde fácilmente el interés por mejorar el bienestar de los terceros en las organizaciones que así lo requieren.

El Anexo 01 contiene la auditoría completa según los lineamientos mínimos descritos en el MGC. Es importante que los contratistas sepan que en cualquier momento pueden ser auditados, no sólo con respecto a los conceptos de SST, sino también con respecto a los subprocesos que comprenden la gestión de contratistas descrita en el Anexo 01.

Para futuras investigaciones, se recomienda ampliar la presente investigación, considerando las variables económicas y financieras de las empresas contratistas y el cliente que son impactadas por el costo de las lesiones y enfermedades ocupacionales de terceros por la falta de gestión de los mismos.

Dependiendo del grado de responsabilidad y de lo hallado en las investigaciones, los incidentes con lesión o accidentes de trabajo suponen un aumento en las primas del seguro, demandas, juicios, pagos de compensaciones, sanciones administrativas y penales para los representantes legales de la empresa. Sin embargo, una de las "sanciones" más importantes no es impuesta por el Estado, sino por las personas involucradas en el accidente de trabajo. La historia no termina con saber si el lesionado se recuperó o si su salud o condición física quedará permanentemente afectada, pues la persona que sufrió la lesión, sea contratista o no, tiene familia y amigos. Los gerentes, deben tomar conciencia de todas las personas afectadas en un incidente; del mismo modo, deben tener especial cuidado en la imagen social que dejan a la comunidad, sobre todo por el cuidado y bienestar de sus colaboradores, ya sean contratistas o trabajadores directos.

\section{REFERENCIAS BIBLIOGRÁFICAS}

[1] Diario Gestión. (27 de abril de 2018). Outsourcing: $86 \%$ de empresas en Perú tercerizan servicios. Recuperado de https:// gestion.pe/economia/outsourcing-86- empresas-peru-tercerizan-servicios-232422noticia/?ref=gesr

[2] Diario Gestión. (15 de septiembre de 2015). Ocho de cada diez empresas en el Perú tercerizan y son $90 \%$ más productivas. Recuperado de https://archivo.gestion.pe/ economia/ocho-cada-diez-empresas-perutercerizan-y-son-90-mas-productivas-2142885

[3] Hernández, R., Fernández, C., y Baptista, P. (2010). Metodología de La Investigación. México D.F., México: McGraw-Hill.

[4] Instituto Sindical de Trabajo, Ambiente y Salud. (s.f.). Salud laboral. Recuperado de https:// istas.net/salud-laboral

[5] International Labour Organization. (6-15 de octubre de 1998). Statistics of occupational injuries. Sixteenth International Conference of Labour Statisticians. Recuperado de https://www.ilo.org/wcmsp5/groups/ public/---dgreports/---stat/documents/ meetingdocument/wcms_088373.pdf

[6] International Labour Organization. (2020). Quick Guide on sources and uses of statistics on occupational safety and health. Switzerland: International Labour Organization. Recuperado de https://ilo.org/wcmsp5/groups/public/--dgreports/---stat/documents/publication/ wcms_759401.pdf

[7] Martínez, R., Tuya, L., Martínez, M., Pérez, A., y Cánovas, A. (2009). El Coeficiente de Correlacion de Los Rangos de Spearman. Revista Habanera de Ciencias Médicas, 8(2). Recuperado de http://ref.scielo.org/842jks

[8] Ministerio de Energía y Minas. (marzo de 2020). La minería peruana en modo COVID-19. Actualización estadística a marzo de 2020. Recuperado de https:// www.minem.gob.pe/minem/archivos/file/ Mineria/PUBLICACIONES/VARIABLES/2020/ BEMMAR20.pdf

[9] Ministerio de Trabajo y Promoción del Empleo. (2017a). Anuario Estadístico Sectorial. Recuperado de http://www2. trabajo.gob.pe/archivos/estadisticas/anuario/ Anuario_2016_020717.pdf

[10] Ministerio de Trabajo y Promoción del Empleo. (2017b). Ley de Seguridad y Salud en el Trabajo, su Reglamento y Modificatorias. Edición Concordada. Recuperado de https://cdn. www.gob.pe/uploads/document/file/349382/ LEY_DE_SEGURIDAD_Y_SALUD_EN_EL_ TRABAJO.pdf 
[11] Minitab. (2019). ¿Qué es estadística descriptiva y estadística inferencial? Recuperado de https://support.minitab.com/es-mx/minitab/18/ help-and-how-to/statistics/basic-statistics/ supporting-topics/basics/what-are-descriptiveand-inferential-statistics/

[12] Organización Panamericana de la Salud. (2020). Salud de los Trabajadores: Recursos Preguntas Frecuentes. Recuperado de https:// www3.paho.org/hq/index.php?option=com_co ntent\&view=article\&id=1527: workers-health-re sources\&ltemid=1349\&limitstart=2\&lang=es
[13] Shapiro, S., y Wilk, M. (1965). An analysis of variance test for normality (complete samples). Biometrika 52, (3-4): 591-611.

[14] Zar, J.H. (1984) Biostatistical Analysis. 2nd Edition, Prentice-Hall, Inc., Englewood Cliffs. 


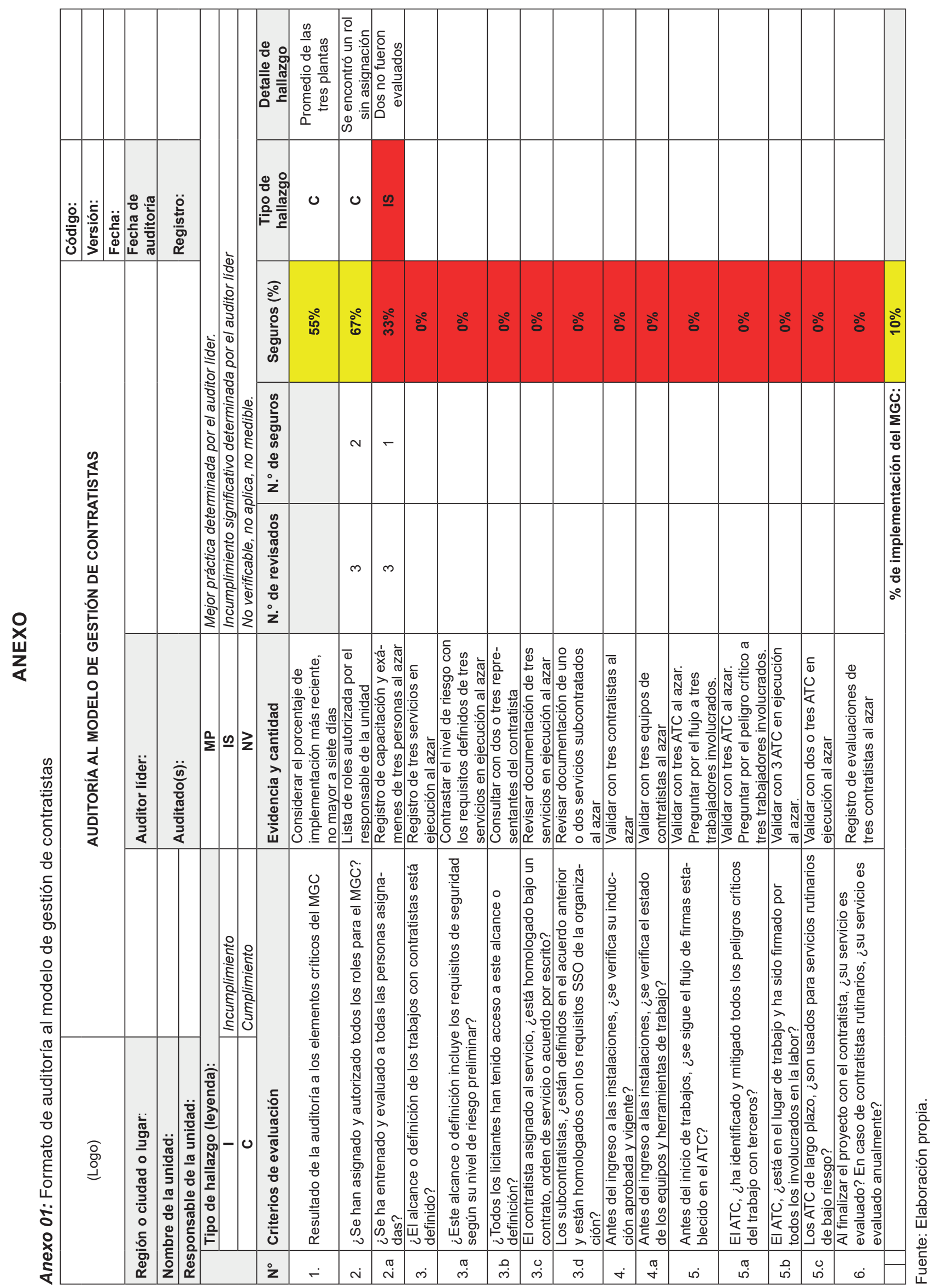




\section{Contractor Management Model to Improve Safety and Productivity Conditions in an Industrial Company}

Franco Rojas Albán ${ }^{1}$

\begin{abstract}
Outsourcing contributes to business development. On some occasions, in order to secure low-cost bids, contractors agree to work under unsafe conditions; on other occasions, the client exerts pressure so that the service is completed ahead of schedule, causing contractors to take "shortcuts" and evade safety controls, which increases the risk of serious injury, chronic illness or even death. In Peru, there is no contractor management model in place to improve safety conditions and promote third-party productivity. Via statistical tests and correlation of variables, this research has developed and implemented a management model that describes the minimum requirements that must be met to avoid incidents and improve contractor productivity. It includes regulations from recognized national and international agencies and encompasses the best work practices acquired in more than 30 years of experience in the management of incidents in manufacturing, construction, steelmaking and mining.
\end{abstract}

Keywords: contractor management; third-party work safety; contractor productivity.

\section{INTRODUCTION}

This research provides a management model designed for service providers (contractors) and organizations that contract services from third parties (clients). This model is designed to:

- protect contractor personnel from injury and illness caused by client activities

- safeguard the assets of both parties involved

- avoid loss of productivity due to injuries or illnesses of contractor workers

- avoid cost inflation and project or service lead times

- improve contractor welfare, social image, public opinion and trust in the marketplace

- clarify the legal responsibility before labor accident investigations or public audits

- prevent the increase of accident rates and insurance cost overruns

- continuously improve performance and promote a safe work culture

This research is relevant and novel for the Peruvian manufacturing sector, since no standard or model exists for managing the safety and productivity of contractors that goes beyond the requirements of Peruvian legislation on outsourced work. The objective of this article, as well as its hypothesis, is to demonstrate that the implementation of a contractor management model (CMM) improves the safety conditions and productivity of third-party contractors in an industrial sector company.

This study was conducted in a metal-mechanical company in the manufacturing sector, which turns steel into consumables for the mining industry. Such company has three industrial plants distributed throughout Peru. For security and confidentiality reasons, the name of the company and the final products will not be disclosed;

1 Industrial Engineer from Universidad Nacional de San Agustín de Arequipa. Currently working as Safety Engineer at Yura S.A. (Arequipa, Peru). ORCID: https://orcid.org/0000-0001-8628-4793 E-mail: frojas87@gmail.com 
however, for the purposes of this research, the company will be referred to as RODAS S.A.

The results of this study can be extended to other organizations in the manufacturing sector, given the great similarity in the way the industry's activities were managed during the study period. Such similarity, in terms of outsourcing and occupational accidents in the sector, is shown in the following paragraphs.

In 2018, the Asociación de Empresas de Tercerización y Trabajo Temporal del Perú (AETT Perú) and Corporativo Overall reported that 8 out of 10 companies in Peru opted for outsourcing services (Diario Gestión, 2018), among which are: recruitment, food, payroll, transportation, surveillance, cleaning, gasfitting, masonry, waste disposal, maintenance, construction, etc. "Tres sectores encabezan la tercerización: Minería y Petróleo con el $50 \%$, Servicio Retail y Consumo con el $30 \%$ y Otros [Three sectors lead outsourcing: mining and oil with $50 \%$, retail and consumer service with $30 \%$ and others (agriculture, construction, manufacturing) with 20\%] (Agricultura, Construcción, Manufactura) con el 20\%" (Diario Gestión, 2015, para. 1).

In its Anuario estadístico sectorial 2016, the Ministerio del Trabajo y Promoción del Empleo (MTPE) declared that during the whole year, 20876 occupational accidents were reported, 17084 (81.8\%) of which occurred in Lima and Callao, making it the region with the most cases (MTPE, 2017a). The number of occupational accidents reported to MTPE, however, is an underestimated figure, since many formal companies, but particularly unlicensed ones, fail to report them due to fear of facing serious sanctions imposed by SUNAFIL for non-compliance with safety conditions (Ministerio de Energía y Minas [MINEM], 2020). Table 1 summarizes such statistics for the purpose of this study.

\section{Occupational Safety and Health (OSH)}

Occupational safety and health is a fundamental aspect of decent work, and decent work is safe work. All workers must be safe in their workplaces, they must have the peace of mind of knowing that they are not exposed to risks and hazards. The physical conditions and mental demands of work and the work environment in general have a strong impact on the well-being and living conditions of workers. Occupational accidents and diseases have a significant human, social and economic cost, so we must strive to prevent them by ensuring that all workplaces are safe (International Labour Organization [ILO], 2020).

La salud laboral se construye en un medio ambiente de trabajo adecuado, con condiciones de trabajo justas, donde los trabajadores puedan desarrollar una actividad con dignidad y donde sea posible su participación para la mejora de las condiciones de salud y seguridad [Occupational health is built in an adequate working environment, with fair working conditions, where workers can perform an activity with dignity and where their participation in the improvement of health and safety conditions is possible]. (Instituto Sindical de TRabajo, Ambiente y Salud [ISTAS], 2008, para. 1)

According to the ILO and the WHO, occupational health is

the promotion and maintenance of the highest degree of physical, mental, and social well-being of workers in all occupations by preventing departures from health, controlling risks and the adaptation of work to people, and the people to their jobs.

Table 1. Comparative Ranking of Occupational Accidents and their Types.

\begin{tabular}{|lll|}
\hline \multicolumn{1}{|c|}{ Accidents by Economic Activity (Top 3) } & $\%$ & \multicolumn{1}{c|}{ Consequences of the Accident } \\
\hline Manufacturing industries & $25 \%$ & Minor accidents \\
\hline Real estate, business and rental activities & $19 \%$ & Disabling accidents \\
\hline Construction & $11 \%$ & Fatal accidents \\
\hline Accidents by Occupation \\
(Top 3)
\end{tabular}

*Including outsourced workers or operators.

Source: Anuario MTPE, 2017. 
(Organización Panamericana de la Salud [OPS], 2020, para. 1)

Occupational health is not limited to safeguarding the physical well-being of the worker, but also deals with the psychological aspect. For employers, occupational health implies support for the improvement of the worker and his or her working capacity (OPS, 2020).

\section{$\mathrm{OSH}$ and Productivity Indicators}

1. Frequency rate of injury incidents (FRII). According to the ILO, this rate is obtained by dividing the number of work-related incidents with injury that occurred during the study period by the number of man-hours worked in the reference group. The ratio is multiplied by an adjustment number, which, for better interpretation, is adjusted according to the magnitude of the hours worked, which can be 100,1000, 100000,1000 000 , etc. (ILO, 2020).

$F R=\frac{\text { Incidents with injury that ocurred during the study period }}{M H} \times 10^{5}$

2. Frequency rate of non-injury incidents (FRNI). This rate is obtained to validate the results presented during the elaboration of the CMM.

$F R=\frac{\text { Incidents with no injuries that ocurred during the study period }}{M H} \times 10^{5}$

It is important to note that man-hours $(\mathrm{MH})$ are obtained from multiplying the number of workers in the reference group by the number of hours worked by them (ILO, 2020). In this case, the reference group is made up of contractor workers.

$M H=$ Number of contractor workers $\times$ Total of worked hours

3. Time available for service (TAS). Similar to the indicators for goods and products, the time available to provide a service has the following formula:

$$
\text { TAS }=\frac{\text { Effective time }}{\text { Available time }} \times 100
$$

Where available time refers to the time allocated by the organization that contracts the service (client) and effective time is the available time minus the time of unscheduled stops due to service interruptions such as incidents, delays in delivery of material, equipment, defective tools, other activities or priorities, etc.

Effective time $=$ Available time - Unscheduled stops

Available time $=$ Time allocated for the service

\section{Contractor Management Model (CMM)}

This model is designed to suit different companies in the industrial sector, be it manufacturing, steelmaking, construction or mining, regardless of their size.

The process starts when the approved work plan for a project or service involves contractors. Figure 1 shows the 4 main sub-processes of the CMM:

Figure 2 shows the stages of the process to establish the occupational safety and health $(\mathrm{OSH})$ conditions necessary to carry out the activities in the approved plan. The level of risk associated with each activity is then identified to obtain the basic and specific requirements, depending on their complexity, so that the organization can bid for the service or project with contractors on equal terms.

Once the proposals are received, they are evaluated (Figure 3). If the contractor has previously worked for the organization, the comprehensive evaluation records are reviewed, a comparative table is drawn up and the contractor with the highest score is selected to undertake the project or service:

Before starting, the selected contractor must go through an induction process to the $\mathrm{OSH}$ culture of the organization, which includes trainings on highrisk jobs required in the plan. After passing the proficiency test, a personal entry credential is issued (see Figure 4).

Once the entry authorization is issued, the rest of the $\mathrm{OSH}$ requirements are reviewed and validated (MTPE, 2017b), such as the complementary risk labor insurance (SCTR in Spanish), the medical clearance, the work tools inspection and other safety documents required by the client. The work authorization process is in charge of the task supervisor assigned by the client. All inspections, observations, occupational accidents or $\mathrm{OSH}$ events are recorded in the CMM (see Figure 5).

Once the work is completed, the contractor is comprehensively evaluated based on the criteria and indicators pre-established in the CMM (see Figure 6). 


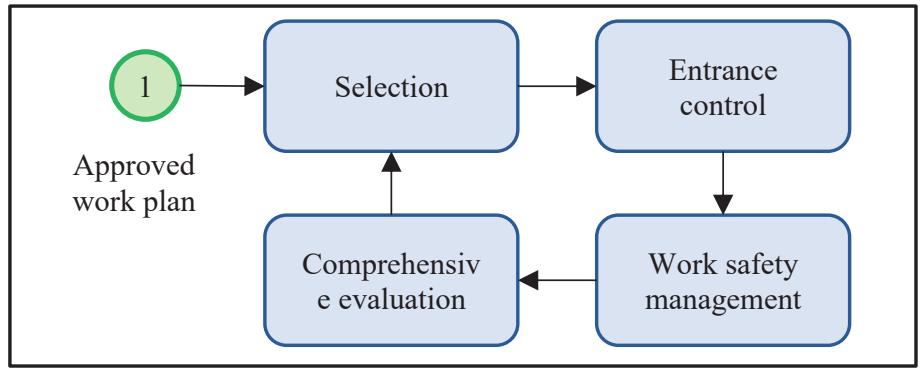

Figure 1. General scheme of the contractor management system. Source: Prepared by the author.

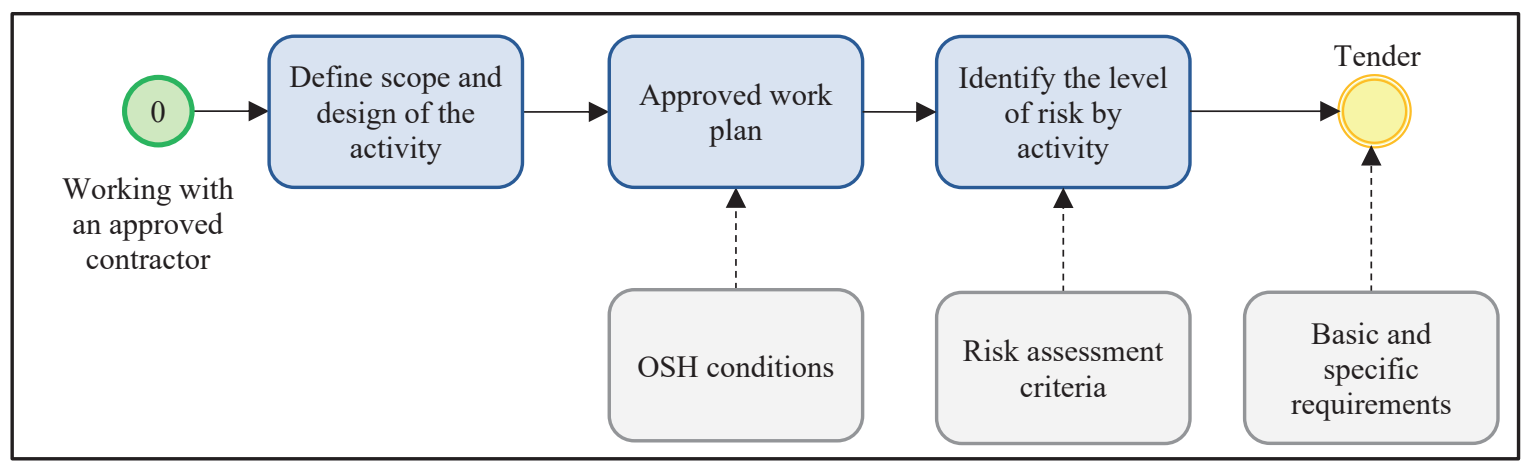

Figure 2. Work plan for contractors.

Source: Prepared by the author.

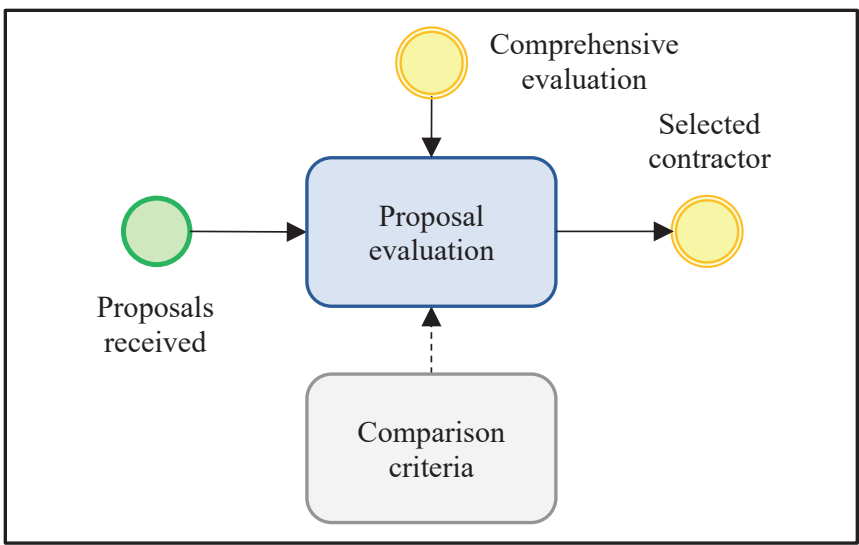

Figure 3. Contractor assessment and selection. Source: Prepared by the author.

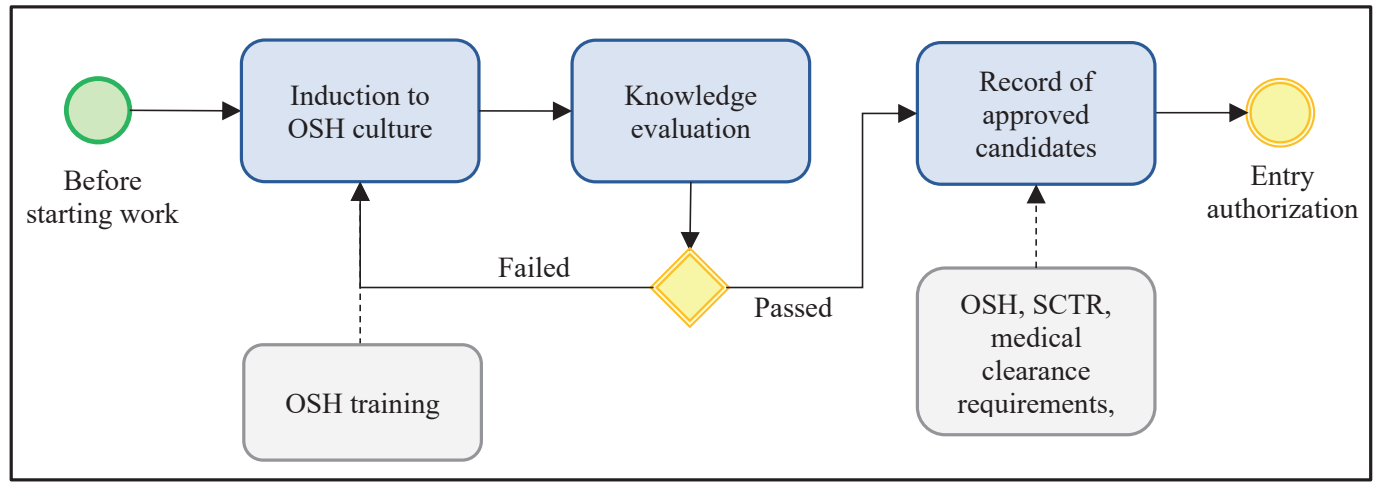

Figure 4. Entrance authorization to the contractor to the work center. Source: Prepared by the author. 


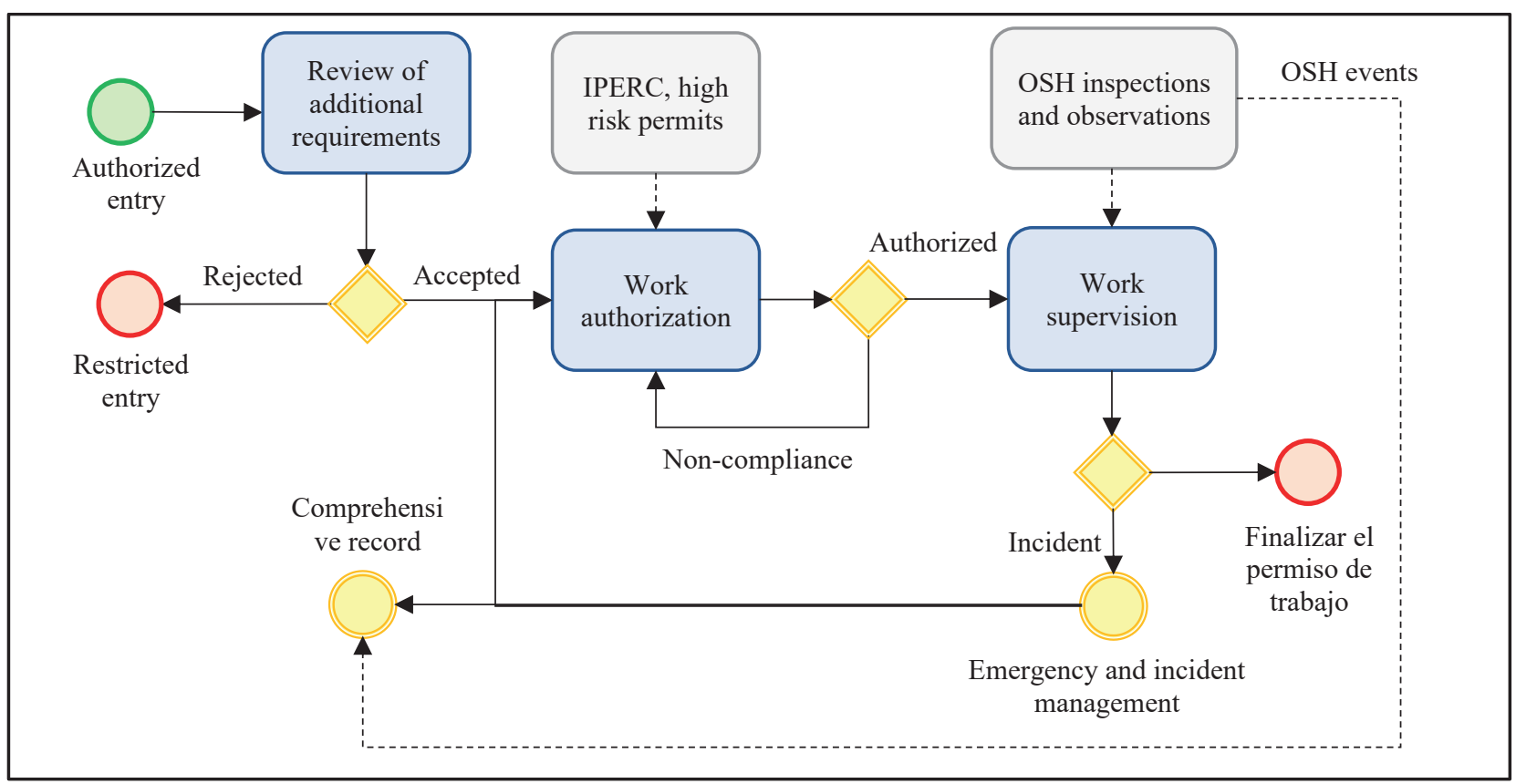

Figure 5. Work safety management.

Source: Prepared by the author.

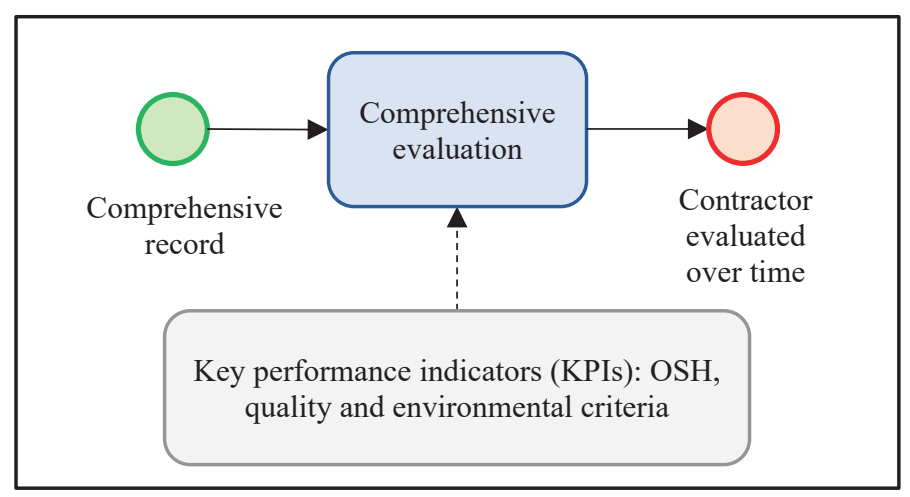

Figure 6. Comprehensive evaluation of the contractor considering $\mathrm{OSH}$, quality and environmental criteria.

Source: Prepared by the author.

\section{METHODOLOGY}

According to the book Metodología de la Investigación by Hernández, Collado, and Baptista (2010), the scope of the present research is correlational, since it aims to determine the relationship or degree of association that exists between the independent variable $\mathrm{X}$, "Implementation of the contractor management model", and two dependent variables: $Y$, "Safety conditions", and Z, "Productivity", in the context of a company in the industrial sector.

The percentage of implementation of the CMM (\% Imp. CMM) was measured quarterly through internal audits. It reflects the percentage of compliance at the time, that may vary over time, but maintains a marked trend. Observed changes in safety conditions (frequency of incidents) and productivity (time available for service) were measured using the units of analysis collected during the period 2012 - 2016.

A causal correlation between variables was demonstrated, that is, that the improvement observed in the dependent variables $Y$ and $Z$ resulted from the improvement in the independent variable $X$. Table 2 shows this relationship.

"Una población es el conjunto de todos los casos que concuerdan con una serie de especificaciones [A population is the set of all cases that meet a set 
of specifications]" (Selltiz, 1980, cited by Hernández, 2010, p. 65). For this study, $100 \%$ of the data collected in the period 2012 - 2016 from the company Rodas S.A. regarding the percentage of implementation of the contractor management model, the number of man hours worked, the incidents of its contractors and the amount of downtime from the incident until the client determined that it was safe to continue were considered.

Data were collected based on the ILO standard and criteria established in the publication "Statistics of Occupational Injuries, Sixteenth International Conference of Labour Statisticians" (ILO, 1998), updated in the "Quick guide on sources and uses of statistics on occupational safety and health" (ILO, 2020). The method used is described in Table 3.

As recommended by the International Labor Organization, fatal and non-fatal incidents were statistically separated in order to avoid biased data and/or values that deviate excessively from the trend and distribution of values (ILO, 2020).

A summary of man-hours and injury and non-injury incidents occurred per year is shown in Table 4.

Table 5 summarizes the downtime per incident per year.

Table 6 summarizes the percentage of implementation of the CMM by year, calculated quarterly using the audit template in Appendix 01:

\section{RESULTS}

An understanding of the descriptive or inferential statistical properties of the resulting data is key to interpreting and evaluating the results. Table 7 shows the summary of the descriptive statistics of the variables' indicators.

Table 2. Unit of Analysis and Measurement.

\begin{tabular}{|l|l|l|}
\hline \multicolumn{1}{|c|}{ Variable } & \multicolumn{1}{|c|}{ Unit of Analysis } & \multicolumn{1}{c|}{ Measurement } \\
\hline $\begin{array}{l}\text { Contractor Management } \\
\text { Model }(\mathrm{X})\end{array}$ & 1. Percentage of implementation & - Quarterly, based on audit results - Appendix 01 \\
\hline Safety conditions $(\mathrm{Y})$ & $\begin{array}{l}\text { OSH indicators: } \\
\text { 2. Frequency rate of injury incidents } \\
\text { 3. Frequency rate non-injury incidents }\end{array}$ & $\begin{array}{l}\text { - Incidents with and without injury } \\
\text { - Number of man-hours worked by contractor } \\
\text { workers }\end{array}$ \\
\hline Productivity $(\mathrm{Z})$ & 4. Time available for service & - Contractor downtime due to incidents \\
\hline
\end{tabular}

Source: Prepared by the author.

Table 3. Data Collection Method.

\begin{tabular}{|c|l|l|}
\hline Var. & \multicolumn{1}{|c|}{ Measurement } & \multicolumn{1}{c|}{ Data Collection Method } \\
\hline $\mathrm{X}$ & $\begin{array}{l}\text { Quarterly, based on audit } \\
\text { results }\end{array}$ & $\begin{array}{l}\text { Documentation, field observations, interviews and surveys that can be verified are required to } \\
\text { carry out the audit of the CMM. Appendix 01 contains a sample of this audit. }\end{array}$ \\
\hline Y & $\begin{array}{l}\text { Number of man-hours } \\
\text { worked by contractor } \\
\text { workers and injury and } \\
\text { non-injury incidents }\end{array}$ & $\begin{array}{l}\text { Measurement, analysis and evaluation of } 100 \% \text { of the data collected during the period } 2012 \\
-2016 \text { in the company Rodas S. A. is required. As this is a strictly quantitative analysis of varia- } \\
\text { bles, no interviews or surveys are required. }\end{array}$ \\
\hline Z & $\begin{array}{l}\text { Contractor downtime due } \\
\text { to incidents }\end{array}$ & $\begin{array}{l}\text { Measurement, analysis and evaluation of 100\% of the data collected during the period 2012 - } \\
2016 \text { in the company Rodas S. A. is required. }\end{array}$ \\
\hline
\end{tabular}

Source: Prepared by the author.

Table 4. Number of Contractor Worker Incidents per Year.

\begin{tabular}{|c|c|c|c|c|}
\hline Year & $\begin{array}{c}\text { Contractor workers } \\
\text { (max.) }\end{array}$ & $\begin{array}{c}\text { Man-hours } \\
\text { (max.) }\end{array}$ & Non-injury incidents & Injury incidents \\
\hline 2012 & 273 & 78656 & 2 & 23 \\
\hline 2013 & 325 & 86625 & 5 & 19 \\
\hline 2014 & 362 & 101424 & 16 & 4 \\
\hline 2015 & 427 & 119236 & 25 & 5 \\
\hline 2016 & 553 & 146739 & 17 & 60 \\
\hline \multicolumn{2}{|r|}{ Total incidents } \\
\hline
\end{tabular}

Source: Prepared by the author based on information provided by the company. 
Table 5. Summary of Service Downtime by Year.

\begin{tabular}{|c|c|}
\hline Year & Service downtime (hrs) \\
\hline 2012 & 170.38 \\
\hline 2013 & 165.73 \\
\hline 2014 & 66.93 \\
\hline 2015 & 25.08 \\
\hline 2016 & 33.41 \\
\hline Grand total & $\mathbf{4 6 1 . 5 3}$ \\
\hline
\end{tabular}

Source: Prepared by the author based on information provided by the company.

Table 6. Summary of the Percentage of Implementation of the CMM

\begin{tabular}{|c|c|}
\hline Year & Implementation of the CMM (\%) \\
\hline $\mathbf{2 0 1 2}$ & $7 \%$ \\
\hline $\mathbf{2 0 1 3}$ & $15 \%$ \\
\hline $\mathbf{2 0 1 4}$ & $87 \%$ \\
\hline $\mathbf{2 0 1 5}$ & $99 \%$ \\
\hline $\mathbf{2 0 1 6}$ & $95 \%$ \\
\hline
\end{tabular}

Source: Prepared by the author.

Table 7. Descriptive Statistics of the Variables' Indicators.

\begin{tabular}{|l|c|c|c|}
\hline & FRNI & FRII & TAS \\
\hline Mean & 1.32 & 1.67 & 7.69 \\
\hline Median & 1.51 & 1.19 & 4.37 \\
\hline Mode & 0.00 & 0.00 & 0.00 \\
\hline Standard deviation & 1.15 & 1.83 & 9.22 \\
\hline Sample variance & 1.32 & 3.35 & 85.07 \\
\hline Kurtosis & -1.05 & 0.62 & 4.94 \\
\hline Skewness coefficient & 0.29 & 1.10 & 1.84 \\
\hline
\end{tabular}

Source: Prepared by the author.

Median and mean values are close in the case of FRNI and FRII; however, mode values, for all the other indicators, suggest that the distribution is probably not normal. A Shapiro Wilk test, used to test the normality of a data set (Shapiro \& Wilk, 1965), was applied to corroborate that the data are non-uniformly distributed. The null hypothesis $\left(\mathrm{H}_{0}\right)$ is that a sample $x_{1}, \ldots, x_{n}$ comes from a population with normal distribution (see Table 8).

Standard deviation and variance values of IFIS show that the dispersion is close; in contrast, the remaining variables exhibit high variability for the averages with respect to the sample trend. The kurtosis and skewness coefficient give an idea of how the distribution curve is formed, and the prominence and symmetry of its tails (Minitab, 2019).

Based on the results of the normality testing, the Spearman correlation test, which is applied to evaluate relationships involving ordinal variables with nonlinear distributions (Zar, 1984), was used.

Figure 7 shows a summary of the incidents that occurred per year to compare the evolution between incidents with injury and without injury, and their interaction with the implementation of the CMM. In the first two years of the study, the number of incidents with injury is significantly high with respect to the percentage of implementation of the CMM; by contrast, the opposite occurs in the last two years of the study. Thus, an inverse or negative proportional relationship exists between the variables.

Figure 8 depicts the annual average of the FRNI in order to compare its evolution over time with respect to the percentage of implementation of the CMM. In the first two years of the study, both the FRNI and the $\mathrm{CMM}$ implementation percentage are significantly low; on the contrary, they are significantly high in the 
Table 8. Normality Testing.

\begin{tabular}{|c|c|c|c|c|}
\hline Indicator & Shapiro-Wilk $(\mathbf{W})$ & $\begin{array}{c}\boldsymbol{p} \text {-value } \\
(\mathbf{0 . 0 5})\end{array}$ & Testing $\left(\mathbf{H}_{\mathbf{0}}\right)$ & Result \\
\hline$\%$ Imp. CMM & 0.7324 & $3.94 \mathrm{e}-09$ & Rejected & Non-normal distribution \\
\hline FRNI & 0.8901 & $5.82 \mathrm{e}-05$ & Rejected & Non-normal distribution \\
\hline FRII & 0.8473 & $2.54 \mathrm{e}-06$ & Rejected & Non-normal distribution \\
\hline TAS & 0.7852 & $5.88 \mathrm{e}-08$ & Rejected & Non-normal distribution \\
\hline
\end{tabular}

Source: Prepared by the author.

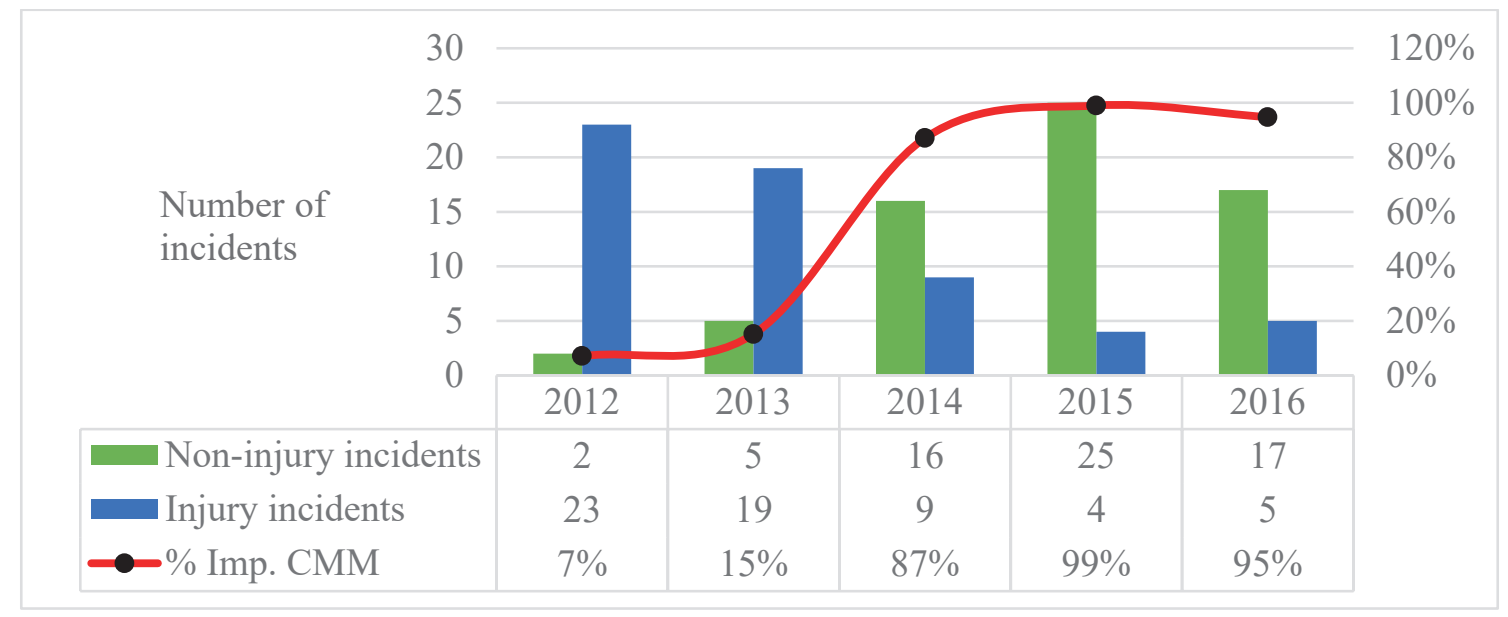

Figure 7. Incident evolution and implementation of the MCC.

Source: Prepared by the author.

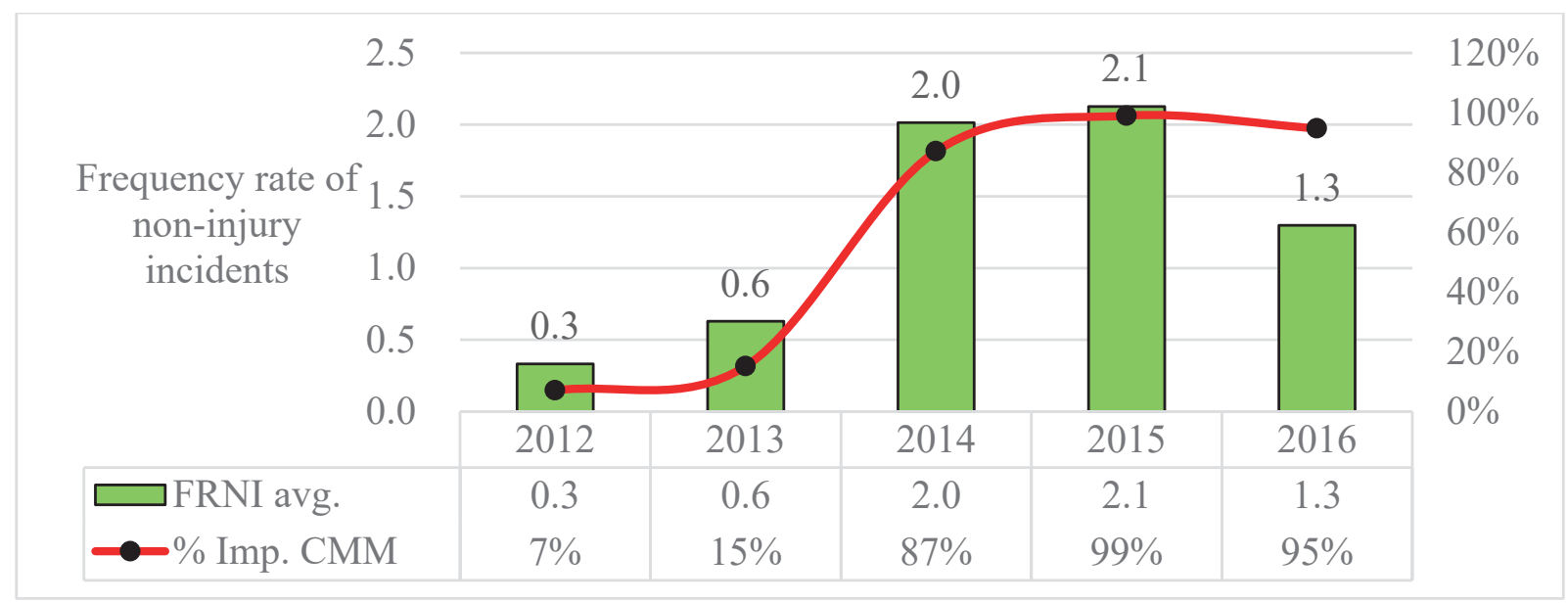

Figure 8. FRNI evolution and CMM implementation percentage.

Source: Prepared by the author.

following two years. Thus, a direct or positive proportional relationship exists between the variables.

Figure 9 depicts the annual average of the FRII in order to compare its evolution over time with respect to the percentage of implementation of the CMM. In the first two years of the study, the FRII is significantly high and the CMM implementation percentage is low; on the contrary, the opposite occurs in the last two years of the study. Thus, an inverse proportional relationship exists between the variables.

Figure 10 shows the annual average of the TAS in order to compare its evolution over time with respect to the percentage of implementation of the CMM. Throughout all the years of the study, both the TAS 


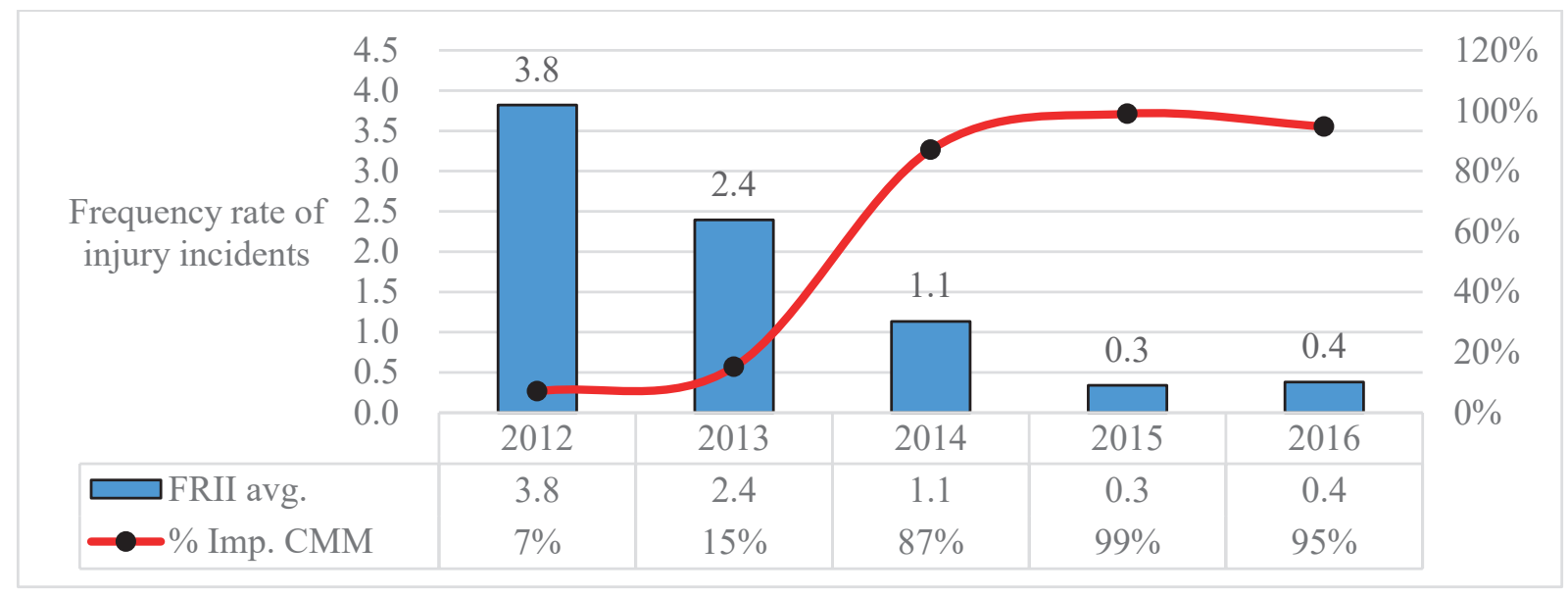

Figure 9. FRII evolution and CMM implementation percentage.

Source: Prepared by the author.

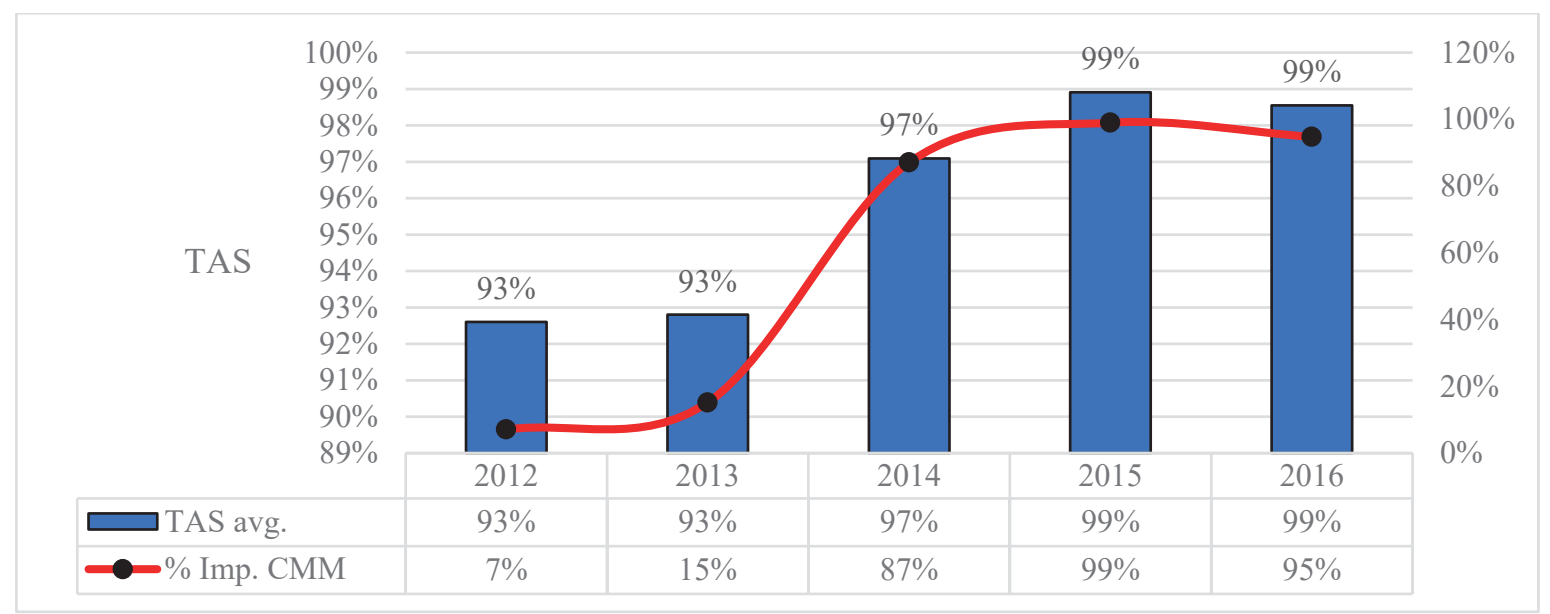

Figure 10. Time available for the service and CMM implementation percentage.

Source: Prepared by the author.

and the percentage of implementation of the CMM vary proportionally over time. Thus, a direct or positive relationship exists between the variables.

\section{DISCUSSION}

Spearman's rating scale presented in Table 9 was used to interpret the results and contrast them with their respective hypothesis (evaluation); the result of this correlation is shown in Table 10.

From the hypothesis testing, it was found that the implementation of a contractor management model has a moderate and strong influence on the safety conditions and productivity of contractors in a company in the industrial sector.
This assertion is supported by the Spearman correlation results obtained for the indicators of the Safety conditions variable which indicate that $63 \%$ of the FRNI is directly explained by the percentage of implementation of the CMM; while $71 \%$ of the FRII is inversely explained by the percentage of implementation of the CMM. For the productivity indicator, $58 \%$ of the TAS is directly explained by the implementation percentage of the CMM. Thus, a moderately strong correlation between variables of the general hypothesis is observed.

\section{CONCLUSIONS}

The research objective was achieved. Through correlation testing, it was demonstrated that the 
Table 9. Spearman's Rho.

\begin{tabular}{|c|c|}
\hline Range & Strength \\
\hline $0.00-0.25$ & Little to none \\
\hline $0.26-0.50$ & Weak \\
\hline $0.51-0.75$ & Moderate to strong \\
\hline $0.76-1.00$ & Strong to perfect \\
\hline
\end{tabular}

Source: Rangos de Spearman (Martínez, Tuya, Martínez, Pérez y Cánovas, 2009).

Table 10. Correlation Between the Variables and the CMM implementation.

\begin{tabular}{|l|c|c|c|c|}
\hline \multicolumn{1}{|c|}{ Variable } & Indicator & Relationship & Rho & Interpretation \\
\hline \multirow{2}{*}{ Safety conditions } & FRNI & Direct & 0.63 & Moderate to strong \\
\cline { 2 - 5 } & FRII & Inverse & 0.71 & Moderate to strong \\
\hline Productivity & TAS & Direct & 0.58 & Moderate to strong \\
\hline
\end{tabular}

Source: Prepared by the author.

implementation of a contractor management model in a company in the industrial sector has had and continues to have a positive impact on the improvement of occupational safety conditions for its contractors.

As shown in Figure 7, through data analysis and the application of correlation testing between variables, it was demonstrated that the CMM implemented at $60 \%$ has a significant impact on the frequency rate of contractors' injuries.

It has been demonstrated that the implementation of the CMM improves the productivity of contractor companies in terms of the availability of time to perform the service by avoiding service interruptions due to the occurrence of injury incidents.

The risk of performing an activity with contractor personnel influences inversely proportional to the level of implementation of the contractor management model, this means that the risk will be high if the contractor management is deficient.

A positive correlation exists between the contractor management model and the frequency of non-injury incidents, since the model encourages and motivates the workers themselves, contractors or not, to report all incidents before encountering dangerous situations that can cause permanent or fatal injuries.

Finally, it can be concluded that the implementation of a contractor management model improves safety and productivity conditions in a company of the industrial sector.

\section{RECOMMENDATIONS}

It is important that senior management and managers understand, are trained and are motivated to implement $100 \%$ of the management model, for without the allocation of personnel and resources, focus on improving the well-being of third-party employees in the organizations that require it is easily lost.

Appendix 01 contains the complete audit in accordance with the minimum guidelines described in the CMM. Contractors should be aware that they can be audited at any time, not only on OSH notions, but also on the sub-processes that comprise the contractor management described in Annex 01.

For future research, this study should be expanded by considering the economic and financial variables of the contractor companies and the client that are impacted by the cost of third party occupational injuries and illnesses due to their poor management.

Depending on the degree of responsibility and the findings of investigations, incidents involving injuries or accidents at work result in increased insurance premiums, claims, lawsuits, judgments, compensation payments, administrative and criminal penalties for the legal representatives of the company. Nonetheless, the most significant "sanction" is imposed by the people involved in the accident. The problem does not end once we know if the injured person recovered or if his/her health or physical condition will be permanently affected, because the person who suffered the injury, whether he/she is a contractor or not, has family and friends. Managers must be 
aware of all the people affected in an incident; likewise, they should pay special attention to the social image they convey to the community, especially for the care and welfare of their collaborators, whether they are contractors or direct workers.

\section{REFERENCES}

[1] Diario Gestión. (April 27, 2018). Outsourcing: 86\% de empresas en Perú tercerizan servicios. Retrieved from https://gestion.pe/economia/ outsourcing-86-empresas-peru-tercerizanservicios-232422-noticia/?ref=gesr

[2] Diario Gestión. (September 15, 2015). Ocho de cada diez empresas en el Perú tercerizan y son 90\% más productivas. Retrieved from https:// archivo.gestion.pe/economia/ocho-cada-diezempresas-peru-tercerizan-y-son-90-masproductivas-2142885

[3] Hernández, R., Fernández, C., \& Baptista, P. (2010). Metodología de La Investigación. México D.F., Mexico: McGraw-Hill.

[4] Instituto Sindical de Trabajo, Ambiente y Salud. (s.f.). Salud laboral. Retrieved from https:// istas.net/salud-laboral

[5] International Labour Organization. (6-15 de octubre de 1998). Statistics of occupational injuries. Sixteenth International Conference of Labour Statisticians. Retrieved from https://www.ilo.org/wcmsp5/groups/ public/---dgreports/---stat/documents/ meetingdocument/wcms_088373.pdf

[6] International Labour Organization. (2020). Quick Guide on sources and uses of statistics on occupational safety and health. Switzerland: International Labour Organization. Retrieved from https://ilo.org/wcmsp5/groups/public/--dgreports/---stat/documents/publication/ wcms_759401.pdf

[7] Martínez, R., Tuya, L., Martínez, M., Pérez, A., \& Cánovas, A. (2009). El Coeficiente de Correlacion de Los Rangos de Spearman. Revista Habanera de Ciencias Médicas, 8(2). Retrieved from http://ref.scielo.org/842jks
[8] Ministerio de Energía y Minas. (March, 2020). La minería peruana en modo COVID-19. Actualización estadística a marzo de 2020. Retrieved from https://www. minem.gob.pe/minem/archivos/file/Mineria/ PUBLICACIONES/VARIABLES/2020/ BEMMAR20.pdf

[9] Ministerio de Trabajo y Promoción del Empleo. (2017a). Anuario Estadístico Sectorial. Retrieved from http://www2. trabajo.gob.pe/archivos/estadisticas/anuario/ Anuario_2016_020717.pdf

[10] Ministerio de Trabajo y Promoción del Empleo. (2017b). Ley de Seguridad y Salud en el Trabajo, su Reglamento y Modificatorias. Edición Concordada. Retrieved from https://cdn. www.gob.pe/uploads/document/file/349382/ LEY_DE_SEGURIDAD_Y_SALUD_EN_EL_ TRAB̄AJŌ.pdf

[11] Minitab. (2019). ¿Qué es estadística descriptiva y estadística inferencial? Retrieved from https://support.minitab.com/es-mx/minitab/18/ help-and-how-to/statistics/basic-statistics/ supporting-topics/basics/what-are-descriptiveand-inferential-statistics/

[12] Organización Panamericana de la Salud. (2020). Salud de los Trabajadores: Recursos Preguntas Frecuentes. Retrieved from https:// www3.paho.org/hq/index.php?option=com_co ntent\&view=article\&id=1527: workers-health-re sources\&ltemid=1349\&limitstart=2\&lang=es

[13] Shapiro, S., \& Wilk, M. (1965). An analysis of variance test for normality (complete samples). Biometrika 52, (3-4): 591-611.

[14] Zar, J.H. (1984) Biostatistical Analysis. 2nd Edition, Prentice-Hall, Inc., Englewood Cliffs. 


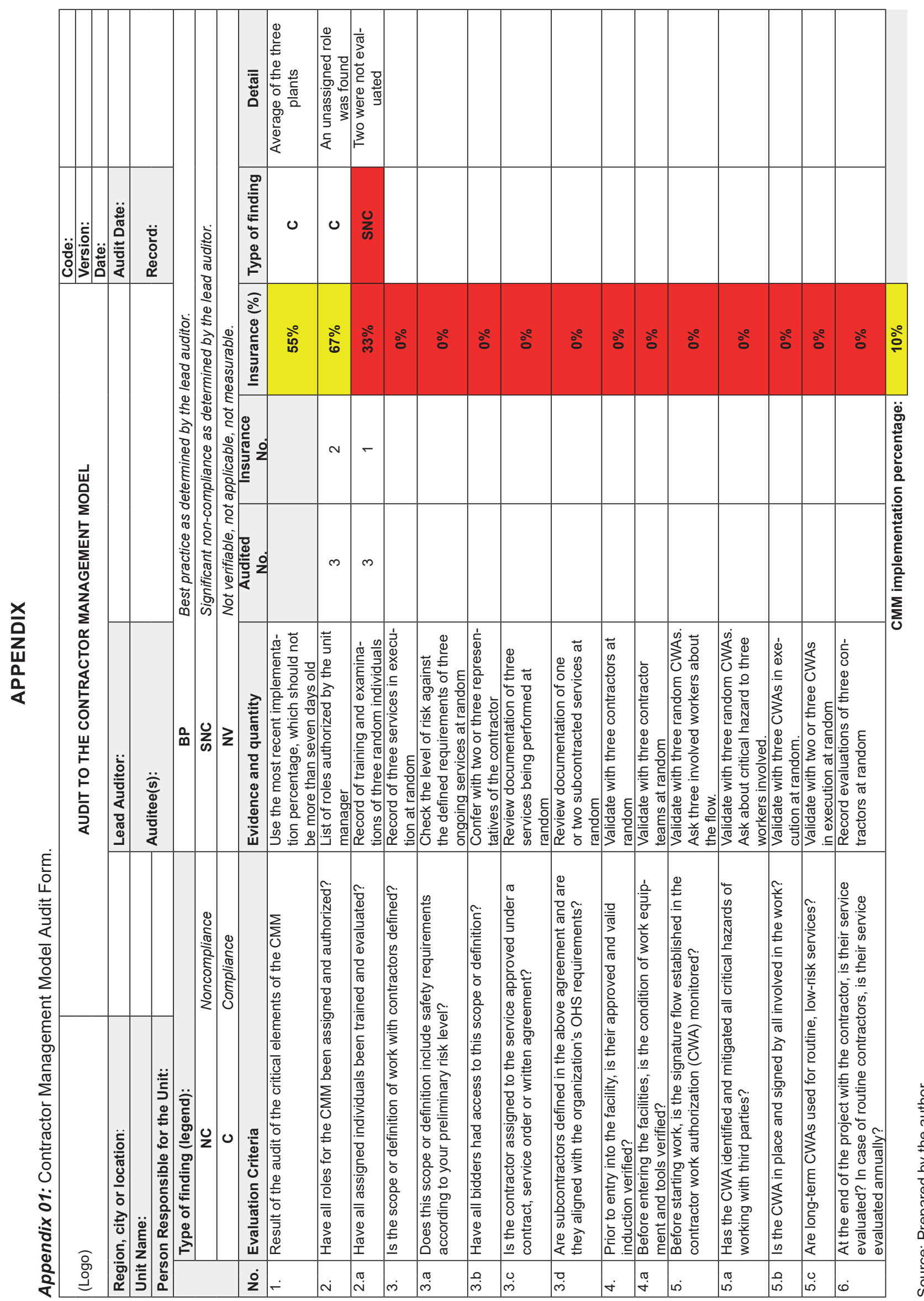

